\title{
Konjunktiv II-Variation im urbanen Sprachgebrauch in Österreich*
}

\author{
Stefanie Edler (Graz) und Georg Oberdorfer (Marburg)
}

\begin{abstract}
The city as a social structure has a distinct relationship with its inhabitants. It creates a sociopragmatic environment that produces communicative characteristics in the form of usage patterns that go hand in hand with conventionalised practices (cf. Gal 1987) and are dependent on everyday-life contextual aspects.

Accordingly, research of urban varieties is ideally based on language data that is as authentic as possible and includes socio-demographic and diatopic aspects. Following this idea, the study presented examines the subjunctive II in urban Austria by analysing free conversations in formal and informal contexts (interviews and conversations among friends), and correlating the results with spatial and social parameters. The data used was collected in Vienna and Graz, the two largest cities in Austria, as well as in their respective surrounding areas and was grouped by gender, age and place of residence.

The subjunctive II is realised in synthetic as well as periphrastic forms (cf. Merkle 1993: 6973.; Donhauser 1992; Glauninger 2008). In rural Austria speakers use the entire range of variants (cf. Ziegler/Glantschnig 2013; Breuer/Wittibschlager 2020), while urban speakers exhibit a much narrower range, as we show in our analysis. Striking features in the use of variants can be seen above all in the form of contrasting modes of construction, i. e. synthetic vs. periphrastic realisation. The study reveals that there are hardly any differences between the two urban centres and only minor differences between a city and its surrounding area. However, the inclusion of social parameters provides a more differentiated picture: Here, higher relative frequencies of synthetic forms are found among male and older speakers compared to female and younger speakers. Thus, a preference for more conservative forms among the former contrasts with a preference for more modern (analytical) variants among the latter.
\end{abstract}

\footnotetext{
* Die präsentierte Studie entstand im Rahmen des an der Universität Graz lokalisierten Forschungsprojekts „Wien und Graz - Städte und ihre sprachlichen Strahlkräfte“, welches Teil des Spezialforschungsbereichs (SFB) „Deutsch in Österreich. Variation - Kontakt - Perzeption“ (SFB F060) ist, finanziert vom Fonds zur Förderung der wissenschaftlichen Forschung (FWF). Der SFB wird getragen von der Universität Wien, der Universität Salzburg, der Universität Graz sowie der Österreichischen Akademie der Wissenschaften und umfasst eine Laufzeit von Jänner 2016 bis Dezember 2023.
} 


\section{$1 \quad$ Einleitung}

Die Stadt unterscheidet sich (wenn auch vielmehr graduell denn oppositionell) vom außerstädtischen bzw. ruralen Raum entlang vielerlei (und untereinander vernetzter) Parameter wie Bevölkerungsdichte, Raumordnung, Infrastruktur, Bevölkerungsstruktur, Vernetzung, soziale Distanz vs. Nähe etc. (cf. etwa Löw/Steets/Stoetzer 2008: 13; Hannemann 2019). Dieses sozietale Gepräge lässt den urbanen Raum als Lebens- und damit Kommunikationsraum mit einer besonderen Konfiguration soziopragmatischer Relationen erscheinen.

Aus linguistischer Sicht bedeutet das, dass die Dynamiken urbaner Zentren mit komplexen kommunikativen Strukturen korrelieren, was zu einer sozialräumlichen Ausprägung an Registern (bzw. Sprechstilen) führt, die zugleich bedarfs-, funktions- und sprechergruppenorientiert gebraucht werden (wenn freilich wohl auch meistenteils, ohne auf einer bewussten Wahl zu beruhen). Das Anliegen dieses Beitrags begründet sich in diesem Zusammenhang auf der - zu erhärtenden - Prämisse, dass die Frage nach dem „Who speaks what language to whom and when?" (Fishman 1965) für die Stadt andere Antworten liefern wird als für den ruralen Bereich: In Bezug auf die Gesprächspartner (,who“ „to whom“) wirkt sich die größere Heterogenität der Einwohner aus, das - damit korrelierende - komplexere Netzwerk sozialer Relationen beeinflusst die Vielfalt und Gestalt der Situationen, in denen sich Interaktionen abspielen (,where“), und das Aufeinandertreffen diverser individueller - sowie auch von Gruppen (partiell) geteilter - Repertoires („what language“) prägt die Stadt als Ort innerer wie äußerer Mehrsprachigkeit. Hinsichtlich der sprachinternen Bandbreite des Deutschen in Österreich ist auch für den urbanen Raum das Dialekt-Standard-Spektrum der gesprochenen Sprache in den Blick zu nehmen. Auch wenn davon auszugehen ist, dass der dialektale Bereich hier vergleichsweise unterrepräsentiert ist, ${ }^{1}$ so wird durch die diversen Sprachbiographien der Stadteinwohner, ihre soziale und regionale Herkunft sowie den Einfluss von Einpendlern dennoch die Präsenz einer Bandbreite von Elementen des Spektrums anzunehmen sein.

Die hier präsentierte Studie leistet einen Beitrag zur Sondierung dieses Spektrums für den Konjunktiv II, indem Befunde zu dessen Formen und Vorkommen im urbanen Österreich vorgestellt und diskutiert werden. Die Basis der Untersuchung bilden Daten von Sprechern aus Wien und Graz, den beiden größten Städten des Landes, sowie aus deren Umland, wobei zusätzlich Faktoren wie Geschlecht, Altersgruppe und Ortschaft differenziert werden. Die Analyse legt ihren Fokus primär auf die Stadt als Sprachraum und lotet den Faktor der Urbanität im Hinblick auf die Wahl sprachlicher Mittel - im Konkreten spezifischer Konjunktiv II-Konstruktionen aus.

Aus sprachwissenschaftlicher Perspektive kann die Stadt als geographisch sowie soziologisch definierte Untersuchungssphäre betrachtet werden. In diesem Sinne sind einerseits räumliche Parameter, andererseits soziale Gegebenheiten in Betracht zu nehmen und (während diese

\footnotetext{
${ }^{1}$ Indem Städte gesteigerten und vielfältigeren sozialen Kontakt bedingen, werden hier vermehrt sprachliche Ausgleichsprozesse begünstigt, welche sich im Abbau regional spezifischer Merkmale zeigen (cf. etwa Kerswill/Williams 2002; Kerswill 2003) und in weiterer Folge von diesen urbanen Zentren ausgehend diffundieren können (cf. z. B. Mattheier 1982; Taeldeman 2005). Zur tendenziell stärkeren Nähe zum Standard im städtischen Bereich cf. z. B. auch die diesbezüglichen Ausführungen bei Soukup (2009: 36-39), Glauninger (2010: 182) oder Ziegler/Oberdorfer/Herbert (in print).
} 
freilich nicht voneinander entkoppelt existieren) je nach Fokus eine areal-horizontale oder sozial-vertikale Perspektivierung anzulegen. In diesem Zusammenhang wird der urbane Raum für die Belange der hier präsentierten Untersuchung als primär sozietal gefasste Entität und nur sekundär als geographisch definierter Ortspunkt begriffen. Im Zentrum steht also der Sprachgebrauch im Spannungsfeld von lebensweltlichen, gesellschaftlichen und pragmatisch-funktionalen Bezügen, oder - mit Fishman (1965) gesprochen - die Frage: Welche Sprecher verwenden wann und in welchen Situationen/mit welchen Gesprächspartnern welche spezifischen Konjunktiv II-Formen? Variation als essentieller Aspekt der Kommunikation tritt in diesem Sinne in der Praxis der alltäglichen Interaktion in Form von gebrauchsorientierten Mustern zutage und ist durch konventionalisierte Praktiken bestimmt (cf. hierzu etwa Denison 1971: 157; Hartung 1981: 96; Gal 1987: 287f.). Sprachgebrauch ist somit sozial und situativ determiniert und kann gleichzeitig umgekehrt soziosemiotische Funktionen erfüllen bzw. Situationen (mit)konstituieren. Das Dialekt-Standard-Kontinuum des gesprochenen Deutsch in Österreich (zur Charakterisierung cf. etwa Auer 2005; Ender/Kaiser 2009; Kaiser/Ender 2015; Soukup/Moosmüller 2011) kann in diesem Sinne als Gefüge von Relationen begriffen werden, die sich zwischen sprachlichen Merkmalen (in diesem Fall divergenten Ausprägungen des Konjunktiv II) und außersprachlichen Kontexten aufspannen. ${ }^{2}$

Vor diesem Hintergrund nimmt die soziolinguistische Erforschung urbanen Sprechens idealerweise Konversationsdaten in den Blick, um ihre Erkenntnisse aus Sprache in Interaktion zu beziehen. Dies betrifft dabei freilich nicht nur qualitativ ausgerichtete Analysen von sich in konkreten, individuellen Gesprächssequenzen vollziehenden sprachlichen Handlungen, sondern scheint uns auch für primär variationslinguistisch-quantitativ orientierte Forschungsfragen stimmig (so wie dies etwa bei Lenzhofer 2017 demonstriert wurde): Indem das Vorkommen und die Häufigkeiten von Instanzen einer Variable wie etwa des Konjunktiv II in möglichst authentischen Interaktionen erhoben werden, sollen die betreffenden Ergebnisse die sprachliche Realität in Bezug auf ein jeweiliges Phänomen adäquat repräsentieren. In diesem Sinne basiert die hier diskutierte Studie auf freien Gesprächen in Form von Interviews sowie Freundesgesprächen, die für tendenziell formelle vs. informelle Interaktionskontexte stehen.

Dies ist die ideell-konzeptionelle Grundlage, auf deren Basis die Konjunktiv II-Variation im urbanen Österreich hier diskutiert wird. Dabei hat die Analyse mehrere Dimensionen in den Blick zu nehmen, liegt das Phänomen, innersprachlich betrachtet, doch an der Schnittstelle von Syntax, Morphologie und Lexik (cf. Stöckle 2020) und berührt außersprachlich zudem soziale, demographische, pragmatische und diatopische Faktoren. Dieser Breite an relevanten Aspekten sollen dementsprechend auch die Forschungsfragen Rechnung tragen:

\footnotetext{
2 Die konkreten Ausprägungen dieser Relationen stellen sich dabei in den Dialektgroßräumen Österreichs freilich mit divergenten Charakteristiken dar: „Während für den alemannischen Westen Österreichs von einem mehr (binnen)diglossischen Varietätenkomplex ausgegangen wird, bei dem sich Dialekt und Standard systemlinguistisch wie sozio-pragmatisch gegenüberstehen, wird für den bairisch-österreichischen Sprachraum eine mehr diaglossische Varietätenstruktur angenommen, die sich durch kontinuierliche Übergänge im ,mittleren Bereich (Bellmann 1983) des Varietätenspektrums auszeichnet“ (Lenz 2019: 321).
} 
- Wie stellen sich die Häufigkeiten und Verteilungen der unterschiedlichen Bildungsmuster im urbanen Raum dar?

- Unterscheiden sich die beiden Städte (Graz und Wien) in Bezug auf die betreffenden Formen und Muster?

- Finden sich Unterschiede hinsichtlich der Konjunktiv II-Konstruktionen zwischen Stadt und Umland und fallen dabei vertikale Parameter in Form von Situation und (Beziehung zum) Gesprächspartner ins Gewicht?

- Welche Rolle spielen Alter und Geschlecht als mögliche Einflussfaktoren auf den Konjunktiv II-Gebrauch?

- Können allgemeine Tendenzen im Hinblick auf funktionale sowie verbspezifische Aspekte festgestellt werden?

Diesen Fragen wird im Folgenden nach theoretischen und konzeptionellen Ausführungen, welche die Basis der Untersuchung erläutern, nachgegangen. Ziel ist es, dabei nicht nur dem Formenspektrum des Konjunktiv II sowie der Distribution einzelner Varianten in unterschiedlichen Bereichen und Gruppen auf den Grund zu gehen, sondern auch Erkenntnisse über das sprachliche Profil des urbanen Raums in Österreich zu erhalten. Als ein morphosyntaktisches Phänomen mit - prinzipiell (!) - hohem Potential für Variation soll der Konjunktiv II Hinweise darauf geben, ob und wie eine solche Variationsbandbreite von Sprechern in der Stadt genutzt wird.

Um die Variable eingangs formell abzustecken, wird zunächst der Varianten-Bestand des Konjunktiv II-Spektrums im relevanten Untersuchungsraum besprochen (Kapitel 2), woraufhin der Forschungsstand zum Phänomenbereich beleuchtet wird (Kapitel 3), bevor sich die folgenden Ausführungen der konkreten Analyse widmen. Dabei klärt zunächst die Darstellung der Datenbasis die Untersuchungsgrundlagen sowie die Methodik (Kapitel 4), bevor die Ergebnisse der Studie präsentiert und eine variations- und soziolinguistische Einordnung des Befundes diskutiert werden (Kapitel 5). Eine Zusammenfassung der vorgelegten Auseinandersetzung mit dem Konjunktiv II im urbanen bairisch-österreichischen Raum bildet schließlich den Abschluss (Kapitel 6).

\section{Der Variationsrahmen - zum Formenbestand des Konjunktiv II im bairischen Di- alektraum des Deutschen in Österreich}

Zunächst sind die realisierbaren Ausprägungen des Konjunktiv II als Untersuchungsvariable zu klären. Dabei soll es nicht darum gehen, im Detail formal das Bildungsmuster bzw. das Kategorienparadigma zu erläutern, sondern vielmehr darum, den Rahmen für mögliche Variation und Variationsmuster abzustecken, indem der für den (sozialen und arealen) Untersuchungsraum gegebene, für die präsentierten Analysen relevante Variablenspielraum dargestellt wird.

Dabei ist zuallererst zu klären, wie das Phänomen des Konjunktiv II umgrenzt ist, da die entsprechende Fachliteratur terminologisch hier keine Einheitlichkeit zeigt. So finden sich theoretische Darstellungen, die den Konjunktiv II formalgrammatisch als zusammenfassenden DachBegriff für Konjunktiv Präteritum und Konjunktiv Plusquamperfekt verstehen. Eisenberg (2006) notiert hierzu etwa, ,dass mit KonjI (=Konj Präs, Konj Pf, Konj Fut) und KonjII (=Konj Prät, Konj Pqpf) lediglich die Konjunktive mehrerer Tempora gemeinsam abkürzend benannt werden. KonjI und KonjII sind keine grammatischen Kategorien“" (Eisenberg 2006: 118). 
Dieselbe Auffassung findet sich auch im Grammatischen Informationssystem grammis des IDS (cf. grammis, Eintrag „Konjunktiv II“). Dieser Systematisierung wird nicht überall gefolgt; der Duden etwa verwendet den Begriff Konjunktiv II offensichtlich synonym zu Konjunktiv Präteritum, ohne die Frage der Bedeutungsgleichheit (und demnach der jeweiligen begrifflichen Extension) explizit zu thematisieren (cf. Duden 2016: 443 et passim), was ebenso in vielen empirisch-analytischen Arbeiten der Fall zu sein scheint (cf. etwa Lotze/Gallmann 2009; Ziegler/Glantschnig 2013; Bercko 2018; Breuer/Wittibschlager 2020). Vielmehr wird demgegenüber aber der Begriff Konjunktiv Präteritum bisweilen auch dezidiert abgelehnt, da die entsprechenden Konjunktivformen zwar formal den Stamm des Präteritums verwenden, funktional bzw. semantisch jedoch keineswegs das temporale Merkmal Präteritum aufweisen (cf. Lotze/Gallmann 2009: 226f.; Breuer/Wittibschlager 2020: 137, FN 1), weswegen für die Bezeichnung als Konjunktiv II plädiert wird.

Diese Diskussion ist hier insofern relevant, als die einzelnen Begriffsauslegungen damit ein unterschiedliches Formenspektrum umfassen. Für die vorgenommene Untersuchung wurde unter den eigentlichen formal definierten Konjunktivformen nur jene berücksichtigt, die dem Konjunktiv Präteritum nach der z. B. bei Eisenberg (2006) vorgestellten Gliederung entsprechen, also die morphologisch realisierten synthetischen Formen, welche auf Basis des Präteritalstammes gebildet werden. Jene Formen, die dort zum Konjunktiv Plusquamperfekt gehören, sprich: die analytischen Formen (aus Konj.Prät. der Auxiliare haben bzw. sein plus Part.Perf.), wurden ausgeklammert. Von Konjunktiv II-Formen kann hier demnach nur dann gesprochen werden, wenn der Konjunktiv II kein Oberbegriff ist, sondern synonym zu Konjunktiv Präteritum gebraucht wird. Zwar hat der Begriff Konjunktiv Präteritum im Sinne einer solchen formalen Klarheit sowie der Transparenz des formalgrammatischen Zusammenhangs mit der Präteritumform durchaus Vorteile, doch soll hier dennoch in weiterer Folge von Konjunktiv II gesprochen werden, um sich der wohl gängigeren Diktion - Lotze/Gallmann (2009: 226f.) etwa stellen diesen Terminus auch als den zeitgemäßeren dar - anzupassen.

Neben diese standardnormgemäße synthetische Konjunktiv II-Form stellen sich im Spektrum periphrastische Konstruktionen, die mit würd- oder tät- plus Infinitiv eines jeweiligen Vollverbs gebildet werden. Dabei stellen diese Verbformen nicht bloß die im Konjunktiv II finiten Bestandteile des Verbalkomplexes dar, vielmehr kann ihnen Auxiliar-Status zugemessen werden: Ganz in diesem Sinne argumentiert auch Smirnova, dass mit der vermehrten Verwendung der würd-Konstruktion ,,allmählich ihre ursprüngliche noch im werden verankerte mutative Bedeutung [verblasst]“ (Smirnova 2006: 190), was einhergeht mit dem hohen Grammatikalisierungsgrad von würd-als Auxiliar. Selbiges sollte auch für die Periphrase mit tät-gelten können, sofern diese Konstruktion die gleiche Einsatzbreite aufweist, was für den österreichischen Raum und die Einschränkung auf die hier relevanten Kontexte, durchaus der Fall zu sein scheint.

Zusätzlich ist dabei zu beachten, dass die periphrastischen Konstruktionstypen freilich formal nicht tatsächlich Teil des Konjunktiv-Paradigmas sind, wie es der Titel dieses Beitrags - verkürzt formuliert - suggeriert. Demgemäß gibt auch Eisenberg zu bedenken, dass ,die würdeKonstruktion nicht ins traditionelle Verbparadigma integriert, sondern [...] Ausdruck des Übergangs von synthetischen zu analytischen Verbformen [ist]“ (Eisenberg 2006: 124; Fettdruck/Kursivierung des Originals getilgt/adaptiert, SE/GO). Funktional werden die periphrasti- 
schen Konstruktionen dennoch als äquivalent und mit den synthetischen Formen (sowie gegeneinander) austauschbar begriffen - zumindest in Kontexten des Ausdrucks der Irrealität oder Indirektheit (cf. Fabricius-Hansen 2000: 91). Fabricius-Hansen kommt in dieser Hinsicht zur Conclusio, dass

die irreale würd(e)-Konstruktion sich grundsätzlich kompositionell, als irrealer Konjunktiv des mit der Fügung werd(en) + Infinitiv ausgedrückten „Non-Realis“, erklären [lässt]. Die Fügung werd (en ) + Infinitiv markiert, dass wir aus der Sicht der Sprecherin die Faktenwelt verlassen; und der präteritale Konjunktiv verschiebt die Welt-Origo weiter ins Irreale. Der ,synthetische“ Konjunktiv Präteritum(perfekt) bewirkt dasselbe in einem Interpretationsschritt.

(Fabricius-Hansen 2000: 91; Kursivierung im Original)

Die Periphrase-Konstruktionen werden daher als gleichwertige Varianten der Variable Konjunktiv II begriffen. In diesem Sinne wird hier pragmatisch-diskurserleichternd verkürzend von Konjunktiv II-Variation gesprochen und damit auf das Gesamtspektrum der vorfindlichen Variablenausprägungen referiert.

In dieses Spektrum gliedert sich zusätzlich eine Reihe an Formen ein, welche als stärker dialektal markiert betrachtet werden: jene Formen, welche mit dem - als typisch für die bairischen Dialekte geltenden (cf. Wiesinger 1989: 57; Donhauser 1992: 226; Stöckle 2020) - Flexiv $-a t^{3}$ versehen sind, das als Infix zwischen Stamm und Personalendung eingefügt wird und sowohl in synthetischen als auch in periphrastischen Konstruktionen auftreten kann. Dabei kann die synthetische Form mit -at- mit Präsens- oder Präteritumstamm gebildet werden.

Im dialektalen Bereich des Bairischen findet sich außerdem eine weitere morphologisch-synthetisch gebildete Konjunktiv II-Form, welche starke Verben mit Ablaut markiert. ${ }^{4}$ Ziegler/Glantschnig (2013: 297), welche die einzelnen Konstruktionstypen entlang eines DialektStandard-Spektrums anordnen, stufen diese als die dialektalste Form ein. Merkle attestiert diesem Typ aber schon vor über einem Vierteljahrhundert das allmähliche Aussterben - insbesondere im städtischen Raum (cf. Merkle 1993: 71). So mutet es wenig überraschend an, dass diese Bildungsweise im gesamten stadtsprachlichen Korpus (inklusive der Umland-Daten), so viel sei hier vorweggenommen, kein einziges Mal vorkommt. Diese Form wurde daher für die Analysen aus dem Variablenspektrum ausgenommen. Abschließend lassen sich damit die in der Untersuchung in Betracht genommenen Formen in der folgenden Übersicht zusammenfassen:

\footnotetext{
${ }^{3}$ Das Flexiv wird nach Wiesinger im Südbairischen als $/ p t /$ und im Mittel- und Nordbairischen als $/ p d /$ realisiert (cf. Wiesinger 1989: 57). Obwohl sich Wien und das Wiener Umland im mittelbairischen und Graz und das Grazer Umland im süd-mittelbairischen Übergangsgebiet befinden, wird hier im Text die Schreibung -at- verwendet, da die lautlichen Qualitäten des Konsonanten für die Untersuchung keine Rolle spielen und -at- auch der in den Transkriptionen der Gesprächsaufnahmen, die den Analysen zugrunde liegen, angewandten Konvention entspricht.

${ }^{4}$ Solche Formen mit sehr „hellem“ frontierten [a] sind etwa nahm (v. nehmen), gschah (v. geschehen), gang (v. gehen), fand (v. finden) etc. (cf. Merkle 1993: 71).
} 


\begin{tabular}{|l|l|}
\hline Bildungsform & Beispiel (konstruiert) \\
\hline 1. $\quad$ synthetisch & nähme, machte \\
\hline 2. $\quad$ synthetisch mit - at- & nehmat/nahmat, måchat \\
\hline 3. periphrastisch mit würd- & würd(e) nehmen, würd(e) machen \\
\hline 4. periphrastisch mit würd- +-at- & wurdat nehmen, wurdat måchen \\
\hline 5. $\quad$ periphrastisch mit tät- & tät nehmen, tät måchen \\
\hline $6 . \quad$ periphrastisch mit tät- +-at- & tatat nehmen, tatat måchen \\
\hline
\end{tabular}

Tabelle 1: Übersicht über die berücksichtigten Konjunktivbildungen

Die hier beigegebenen konstruierten Beispiele sollen einen hinreichenden Eindruck der morphosyntaktischen Konstruktionsweisen geben, sie sind freilich nicht geeignet, lautliche Variabilität und das Spektrum möglicher phonetischer Realisierungsalternativen abzubilden. Der damit begangene Mittelweg zwischen standardorthographischer und nonstandard-lautorientierter Notation ist somit nur ein Kompromiss, der darauf abzielt, den Blick auf die für die Analyse relevanten Aspekte zu richten. Die damit abgebildete potentielle Variationsbandbreite stellt den Rahmen dar, in welchem die Variable Konjunktiv II im Sprachgebrauch des urbanen Raums sondiert wird. Nachdem damit die phänomenologische Analysesphäre abgesteckt ist, schließt sich ein Blick auf die Forschungslage mit besonderem Augenmerk auf Abhandlungen zum Konjunktiv II im Kontext von Sprache und Sprechen in der Stadt an, bevor das Untersuchungsdesign zu besprechen ist.

\section{Zum Forschungsstand}

Die Stadt als Kommunikationsraum hat international im Bereich der Sozio- und Variationslinguistik immer eine bedeutende Rolle gespielt. Als gesellschaftlich diverses Konglomerat, gekennzeichnet durch innere und äußere Mehrsprachigkeit (cf. Wandruszka 1979) sowie ,glossotopisch[e] Verflechtung[en]" (Krefeld 2015: 402), hat der urbane Raum nicht nur ein hohes Potential für ein breites Variationsspektrum; darüber hinaus erweist er sich häufig auch als Ort, der Sprachwandeltendenzen anstößt - einerseits werden von ihm ausgehend sprachliche Neuerungen verbreitet (cf. Taeldeman 2005: 267f.; Glauninger 2008: 235), andererseits ist er zugleich aber auch Schauplatz von Homogenisierungen (cf. Kerswill/Williams 2002; Kerswill 2003).

In Österreich wurde einem entsprechenden Forschungsinteresse an der Stadt - zumal im internationalen Vergleich - noch bei weitem nicht in einem Ausmaß Rechnung getragen, welches das Erkenntnispotential einer solchen Perspektivierung ausschöpft, was jedoch freilich nicht das Bestehen und die Qualität der bislang vorliegenden Studien zum Thema in Abrede stellen soll: So finden sich Untersuchungen zu bestimmten Einzelphänomenen unter verschiedenen Perspektiven, etwa zum (er)l-Diminutiv in Graz von Glauninger (2005), Analysen zu spezifischen linguistischen Sprachbeschreibungsebenen, wie im Bereich der Soziophonologie z. B. von Dressler/Wodak (1982) für Wien, von Moosmüller (1991) für Wien, Graz, Salzburg und Innsbruck oder von Moosmüller/Scheutz (2013) für Salzburg und Wien, sowie auch Arbeiten mit Fokus auf bestimmte Gruppen, etwa zur inneren Mehrsprachigkeit Jugendlicher in Wien 
von Glauninger (2010). ${ }^{5}$ Auch bietet Hutterer (1978) eine Überblicksdarstellung zur Sprache in Graz, welche allerdings ihre empirische Grundlage nicht offenlegt und im Allgemeinen einen stark generalisierenden Charakter aufweist.

In Bezug auf den Konjunktiv II im Allgemeinen finden sich neben Kapiteln oder Abschnitten in Grammatiken (z. B. Duden 2016: 550-553; Eisenberg 2006: 116-124) und theoretischsprachstrukturell orientierten Arbeiten (etwa Eisenberg 2005; Gallmann 2007; Lotze/Gallmann 2009; 6 Thieroff 2010; Wiesinger 1989) auch funktional fokussierte Darstellungen (wie Fabricius-Hansen 2000; Glauninger 2011) sowie Studien mit historischem Fokus (insbesondere zur würd-Periphrase von Auer 2007 sowie von Smirnova 2006).

Überblickt man die zum Thema vorliegenden empirisch fundierten Untersuchungen zum Deutschen, so zeigt sich dabei eine Bandbreite an unterschiedlichen Sprachraumbezügen, Datengrundlagen sowie methodischen Ansätzen. Während Lenz (2016) ihre Darstellung für Hessen (im Rahmen des Projekts Syntax hessischer Dialekte $(S y H D)$ ) auf Fragebogendaten sowie mündlichen Übersetzungsaufgaben-Output basiert und Bittner/Köpcke (2010) ihre auf den Schweizer und norddeutschen Raum bezogene Untersuchung auf (teils mündlich, teils schriftlich durchgeführte) Sprecherbefragungen und Lückentextaufgaben gründen, stützt Graf (1977) seine Arbeit, die Baden-Württemberg, Bayerisch-Schwaben, Vorarlberg und Liechtenstein berücksichtigt, auf Konversationsdaten sowie auch Donhauser (1992) ihre Studie zum Bayerischen Wald auf stärker konversationsnahe Aufnahmen in Form von ,authentischen Erzählungen" basiert.

Ferner lassen sich auch sprachgeographische Daten zum Konjunktiv in Sprachatlanten finden, im konkreten Fall insbesondere in den Bayerischen Sprachatlanten (wie dem Sprachatlas von Niederbayern ( $\mathrm{SNiB}$ )), die in den letzten Jahrzehnten entstanden sind (cf. für einen Überblick die Atlantenbeschreibung in REDE und das dort integrierte SprachGIS).

Enger gefasst, mit spezifischem Augenmerk auf den bairisch-österreichischen Raum innerhalb Österreichs, finden sich ebenso unterschiedlich orientierte variationslinguistische Arbeiten zum Konjunktiv II. Während Breuer/Wittibschlager (2020) ihre Analysen auf Experimentdaten stützen und Glauninger (2008) auf Basis von Befragungen arbeitet, beruhen die Untersuchungen von Ziegler/Glantschnig (2013), Lenzhofer (2017) und Bercko (2018) auf Konversationsdaten. Stöckle (2020) dagegen, der eine sprachhistorische Perspektive eröffnet, arbeitet mit der Belegsammlung des WBÖ und gründet seine Analyse auf Handzettel (die größtenteils aus der ersten Hälfte des 20. Jh. stammen). Das Thema wurde also bislang mit recht unterschiedlichen Schwerpunkten und auf Basis sehr verschiedener Datengrundlagen behandelt.

Für den Kontext des vorliegenden Beitrags erweisen sich drei der genannten Arbeiten als in engerem Sinne relevant - insofern als sie in die gleiche Stoßrichtung zielen und ebenso die Konjunktiv II-Variation im urbanen Raum behandeln: Bei Glauningers (2008) Untersuchung, die auf einer Befragung von 27 Wiener Jugendlichen basiert, handelt es sich per Eigendefinition

\footnotetext{
${ }^{5}$ Eine umfassende, empirisch fundierte Analyse der syntaktischen Variation des Deutschen in Wien, die Ansätze der Regionalsprachenforschung mit jenen der Stadtsprachenforschung verquickt, wird darüber hinaus von Breuer (in Vorbereitung) erwartet.

${ }^{6}$ Wobei hier das Formenspektrum des Konjunktivs und seine Variation diskutiert werden, allerdings auf rein theoretischer Ebene.
} 
um eine „narrativ[e] Fallstudie“ (Glauninger 2008: 234), die auf Einsichten zur Rezeption, Produktion und Verwendung unterschiedlicher Konjunktiv II-Konstruktionen abzielt. Hier zeigt sich, dass die Jugendlichen des urbanen Wiens Probleme bei der Dekodierung dialektal-synthetischer Konjunktivformen (mit Ausnahme von Modalverben) haben sowie eine starke Tendenz zu analytischen Varianten und hierunter zu Konstruktionen mit würd- (gegenüber tät-) aufweisen.

Die Untersuchung Berckos (2018) gründet auf Konversationsdaten in Form von Freundesgesprächen aus Graz sowie zwei ruralen Gemeinden. Auch hier stellt sich für die Stadt eine starke Tendenz zu analytisch gebildeten Formen und darunter insbesondere auch deutlich zur würdPeriphrase heraus, während Sprecher in den ruraleren Ortspunkten eher zur tät-Periphrase bzw. noch vielmehr zu synthetischen Formen mit -at-Infix neigen. Zum Gewicht der Untersuchung ist allerdings anzumerken, dass die Ergebnisse wohl eher nur einen vorläufig-explorativen Eindruck geben können, da insgesamt nur 12 Informanten (bzw. in Summe nur 35 Vorkommen der Variable) untersucht wurden.

Breuer/Wittibschlager (2020) stützen sich auf eine umfassendere Quellenlage und integrieren die Daten von insgesamt 138 Informanten (122 aus dem ruralen und 16 aus dem urbanen Bereich $^{7}$ ) in ihre Studie. Sie behandeln sowohl urbane als auch rurale Gebiete in Österreich mit der Bundeshauptstadt Wien als städtischem Ortspunkt, wobei die areal-horizontale Perspektive mit einer sozial-vertikalen zusammengeführt wird. Ihre Analyse stützt sich auf Daten, die in zwei unterschiedlichen Experimentdurchläufen elizitiert wurden: ,a formal situation aiming at more standard-conform varieties and an informal situation for more non-standard/dialectal varieties“(Breuer/Wittibschlager 2020: 146). Hier ergibt sich ebenfalls insgesamt eine Dominanz der würd-Konstruktionen sowie auch eine allgemeine Tendenz zur vermehrten Verwendung analytischer Bildungen bei jungen Sprechern, was dem von Glauninger (2008) aufgezeigten Trend entspricht.

Hinsichtlich der stadtspezifischen Fragestellungen zeigt sich, dass in der Standard-Erhebung die Präferenz der würd-Konstruktion für den urbanen gleichermaßen wie für den ruralen Raum gilt, während an zweiter Stelle synthetische Formen stehen. Auch die Dialekt-Erhebung bestätigt die allgemeine Bevorzugung der Periphrase, zeigt allerdings ein differenzierteres Bild in Bezug auf das dabei verwendete Auxiliar: Im ruralen Raum stellen sich beide Periphrase-Konstruktionen (jene mit tät- zu rund 34\%, jene mit würd- zu 32\%) als stark vertreten heraus; die urbanen Sprecher dagegen geben (mit einem Anteil von rund 47\%) eindeutig der Form mit würd- den Vorzug und gebrauchen selbst die standardnormgemäßen synthetischen Konstruktionen häufiger als die tät-Periphrasen (die mit nur rund 13\% vertreten sind) (cf. Breuer/Wittibschlager 2020: 161). ${ }^{8}$ Wenn in dieser Studie die Gesamtzahl an Vorkommen an Konjunktiv II-

\footnotetext{
${ }^{7}$ Addiert aus Tabelle 2 in Breuer/Wittibschlager 2020: 148.

${ }^{8}$ An früherer Stelle wird dabei im Zusammenhang mit der areal-horizontalen Perspektive auf die Daten zugleich ein (dem anderen Blickwinkel geschuldetes) anderes Bild gezeichnet: Dort fokussieren die Autoren auf areale Variationsmuster und stellen die Häufigkeiten getrennt für die jeweils einzelnen Ortspunkte dar. Werden die Frequenzen in dieser Form perspektiviert, zeigt sich, dass in circa der Hälfte der (ausschließlich ruralen) Erhebungsorte tät- überwiegt, während die andere Hälfte, welche Wien sowie aber auch fünf rurale Ortschaften inkludiert, würd-als präferierte Variante aufweist (cf. Breuer/Wittibschlager 2020: 155f.). In diesem Sinne scheint es angemessen, den Faktor „Urbanität“ ein wenig zu relativieren, zeigt diese Darstellung doch, dass die größere Neigung
} 
Formen auch sehr umfassend ist, so wiegen die Daten des ruralen Bereichs weit mehr, während für den urbanen Bereich nur 59 Instanzen $^{9}$ der Variable in die Analysen einflossen.

Alle drei Untersuchungen kommen zum Ergebnis, dass sich im städtischen Raum eine deutliche Tendenz zu analytischen Bildungstypen zeigt und innerhalb dieser zu Formen mit würd-. Auch wenn damit bereits ein wesentlicher Trend auf empirischer Ebene nachgewiesen und Sprachgebrauchsmuster im Bereich der Konjunktiv II-Verwendung aufgezeigt sind, vermag eine weitere Untersuchung zweifellos einen Beitrag zu einem breiteren und tiefergehenden Erkenntnisstand zur Konjunktiv II-Variation im urbanen Raum Österreichs zu leisten, wobei fallweise bestätigende sowie erweiternde Einsichten komplementiert werden können. Hier spielt zum einen die Erhebungsmethodik eine Rolle: Während Glauninger (2008) und Breuer/Wittibschlager (2020) keine Konversationsdaten im Sinne umfassenderer Gesprächsaufzeichnungen verwenden, liegen der hier präsentierten Studie (zumindest annäherungsweise authentische) freie Gespräche in Form von (leitfadenorientierten) Interviews und Freundesgesprächen zugrunde. Eine ähnliche Erhebungsmethode - basierend auf Freundesgesprächen in Gruppen - verwendet auch Bercko (2018); doch ist hier die Datenbasis nicht sehr aussagekräftig. Und auch bei Breuer/Wittibschlager (2020) ist der urbane Raum weniger stark repräsentiert, während Glauninger (2008) zu seiner Befragung gar keine systematisierten Quantitäten anführt, geht es ihm doch auch um eine etwas andere Zielsetzung, die funktional-pragmatische Aspekte anvisiert. Auch in dieser Hinsicht versucht die hier besprochene Studie ein breiteres Fundament zu bieten, indem für den Stadtraum eine größere Zahl an Konjunktiv II-Instanzen in die Analysen einfließen, welche zumal von den Sprechern ohne direkten Einfluss im Gesprächsverlauf produziert wurden. Es ist deshalb davon auszugehen, dass die damit abgebildeten Verwendungsmuster und -häufigkeiten der Sprachgebrauchsrealität letztlich näher kommen als in indirekteren oder unnatürlicheren Settings erhobene Realisierungen. Zudem wurde durch die Berücksichtigung der beiden Städte Wien und Graz auch eine lokale Erweiterung hinsichtlich des urbanen Raumes gegenüber bestehenden Untersuchungen vorgenommen.

\section{Zur Datenbasis - konzeptionelle und methodische Grundlagen}

Um diese groben Angaben auszuführen, sei im Folgenden das Untersuchungsdesign der Studie genauer beleuchtet. Die Datenbasis der Analyse speist sich aus dem Stadtsprachenkorpus (ULAKorpus) des Forschungsprojekts Wien und Graz - Städte und ihre sprachlichen Strahlkräfte, welches Gesprächsdaten aus Wien (1.911.191 EW) und Graz (291.072 EW) ${ }^{10}$ sowie deren jeweiligem Umland enthält, wobei die betreffenden Gemeinden der urbanen Agglomeration für Wien in Gerasdorf (11.401 EW), Mödling (20.564 EW) und Baden (25.958 EW), für Graz in Weinitzen (2.670 EW), Kalsdorf (7.572 EW) und Thal (2.288 EW) bestehen (siehe Abbildung 1). ${ }^{11}$

\footnotetext{
zum Gebrauch der periphrastischen Konstruktion mit würd-kein städtisches Spezifikum darstellt. Im Kontext der arealen Auswertung heißt es zudem auch: „No significant urban/rural difference could be found.“ (Breuer/Wittibschlager 2020: 159).

${ }^{9}$ Es handelt sich dabei um einen aus den im Beitrag präsentierten Grafiken addierten Wert (cf. z. B. Breuer/Wittibschlager 2020: 153, Grafiken 2 und 3).

${ }^{10}$ Zahlen via Statistik Austria, welche dem Stand zum 01.01.2020 entsprechen (cf. Statistik Austria 1).

${ }^{11}$ Zahlen via Statistik Austria, welche dem Stand zum 1.1.2020 entsprechen (cf. Statistik Austria 2).
} 


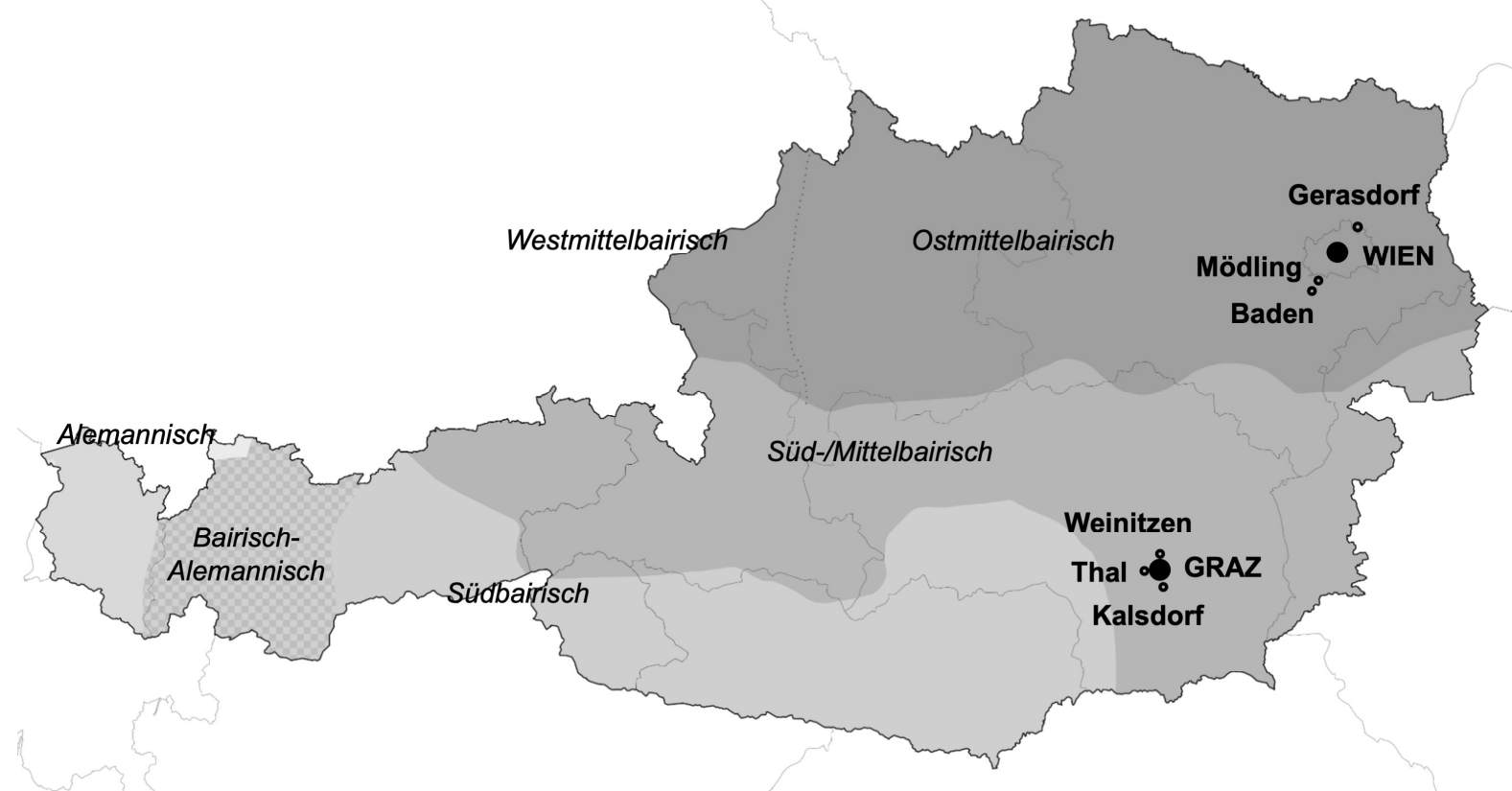
Abbildung 1: Karte von Österreich mit den Erhebungsorten des ULA-Korpus
(CC-BY-SA Melanie Seltmann, dioe.at, regionalsprache.de)

Als zusätzliche Kriterien für die Selektion der Gewährspersonen galten Geschlecht und Alter, wobei ein 1:1-Geschlechterverhältnis angestrebt wurde und die Sprecher in eine ältere Gruppe 65+-Jähriger und eine jüngere Gruppe 20- bis 30-Jähriger unterteilt wurden. Die erhobenen Daten bestehen, wie schon einleitend erwähnt, in Gesprächsaufnahmen in Form von - tendenziell formellen - strukturierten Interviews (geführt von einem für die Gewährspersonen fremden Explorator) sowie - tendenziell informellen - Freundesgesprächen (zu zweit geführt unter vertrauten Personen in Abwesenheit des Explorators).

Die Studie zum Konjunktiv II basiert auf zwei Sub- bzw. Teilkorpora: Korpus A, welches ausschließlich Interviews enthält und 28 Gewährspersonen inkludiert, wobei je sieben aus Wien und aus Graz sowie abermals je sieben aus dem jeweiligen Umland stammen (sodass 14 urbane 14 nicht-urbanen Sprechern gegenüberstehen), und Korpus B, welches sowohl aus Interviews als auch aus Freundesgesprächen besteht und 16 Gewährspersonen inkludiert, wobei sieben Personen Graz, vier dem Grazer Umland, drei Wien und zwei dem Wiener Umland zuzuordnen sind $^{12}$ (siehe Tabelle 2). ${ }^{13}$ Dieser Ungleichgewichtung wird in den Auswertungen Rechnung getragen, indem die Daten nur normalisiert verglichen werden und stets relative Häufigkeiten gegenübergestellt werden. Dass die Aussagekraft in Bezug auf nur durch kleine Zahlen vertretene Subsamples hier nur eine reduzierte sein kann, ist freilich evident; die Perspektive auf die

\footnotetext{
12 Für Korpus A besteht insgesamt ein 1:1-Verhältnis zwischen der jungen und der alten Sprechergruppe, zwischen männlichen und weiblichen Sprechern, zwischen Gewährspersonen aus dem Grazer Großraum und dem Wiener Großraum sowie auch zwischen einerseits allen urbanen Informanten zusammengefasst und andererseits allen nicht-urbanen. Dieses Gleichgewicht konnte bei Korpus B leider nicht gewährleistet werden.

13 Alle Gewährspersonen in Korpus B sind auch in Korpus A vertreten. Korpus B stellt insofern in Bezug auf die Sprecher ein Teilsample von Korpus A dar, ist jedoch erweitert um das zusätzliche Erhebungssetting.
} 
vertikale Ebene soll exemplarisch-explorativ dennoch zusätzliche Hinweise auf den Konjunktiv II-Gebrauch in näherungsweise alltäglichen Interaktionen geben.

\begin{tabular}{|l|l|l|c|}
\hline Teilkorpus & Settings & Erhebungsort & Anzahl der Sprecher \\
\hline \multirow{4}{*}{ Korpus A } & \multirow{2}{*}{ Interviews } & Graz Stadt & 7 \\
\cline { 3 - 4 } & & Graz Umland & 7 \\
\cline { 3 - 4 } & & Wien Stadt & 7 \\
\cline { 3 - 4 } & Wien Umland & 7 \\
\cline { 3 - 4 } & & GESAMT & 28 \\
\hline \multirow{4}{*}{ Korpus B } & \multirow{2}{*}{ Interviews und } & Graz Stadt & 7 \\
\cline { 3 - 4 } & Freundesgespräche & Graz Umland & 4 \\
\cline { 3 - 4 } & & Wien Stadt & 3 \\
\cline { 3 - 4 } & & Wien Umland & 16 \\
\cline { 3 - 4 } & & GESAMT & 2 \\
\hline
\end{tabular}

Tabelle 2: Korpus-Übersicht zu den Erhebungsorten und Sprechern

Die Gesamtgesprächszeit der beiden Korpora beträgt ca. 35 Stunden, in welchen insgesamt 1087 Instanzen der Konjunktiv II-Variable gefunden wurden (620 in Interview-, 467 in Freundesgesprächen), wobei sich diese Vorkommen auf 161 Verblexeme verteilen. Die Datenbearbeitung erfolgte auf Basis von Transkripten der Aufnahmen und in Form von manuellen Annotationen. Berücksichtigt wurden, wie bereits in Kapitel zwei vorweggenommen, ausschließlich Formen des sogenannten Konjunktiv Präteritum, während die analytischen Formen des Konjunktiv Plusquamperfekt ausgeschlossen wurden, um die Gruppe an Varianten funktional homogener zu halten sowie sicherzustellen, dass grundsätzliches Potential der Variation über das gesamte Spektrum gegeben ist. ${ }^{14}$ Ebenso aus der Erhebung ausgenommen wurden sämtliche Vorkommen von möchte, dessen Status als umstritten gilt (cf. etwa Mortelmans/Smirnova 2010: 50, FN 27). Hier folgen wir Lehmann (1995), welcher möchte als Desiderativ-Auxiliar begreift und in Bezug auf den Grammatikalisierungsstatus neben würde stellt: „They only occur in finite forms of what is formally the past subjunctive and are no longer synchronically related to werden 'become' and mögen 'may, like"“ (Lehmann 1995: 141, Kursivierung im Original). Da es demnach nicht nur keine äquivalente Alternation zwischen z. B. möchte und würde mögen gibt, sondern zudem der Konjunktivstatus von möchte mindestens fragwürdig ist, wurde das Lexem grundsätzlich ausgeschlossen. Andere Arbeiten klammern ohnehin die gesamte Gruppe der Modalverben - darunter mögen - aus (cf. etwa Ziegler/Glantschnig 2013: 298, FN 16; Lenzhofer 2017: 282). Auch für den vorliegenden Beitrag wurde ebenjene Gruppe

\footnotetext{
14 Hierzu sei etwa auf Ausführungen zu morphopragmatischen Unterschieden bei Glauninger (2008: 245) verwiesen, welcher auf die Funktion der Sprechaktmodifikation eingeht, die dem Konjunktiv II inhärent sei, von der periphrastischen würd-Konstruktion aber nur partiell erfüllt werden kann - nämlich nur im Konjunktiv Präteritum, nicht aber im Konjunktiv Plusquamperfekt: Dort sind die Formen I hed gsagt/I hed gsogt vs. I wüad gsagt habn/ I wüad gsogt hobn (Beispiele übernommen von Glauninger 2008: 245) nicht funktional bzw. pragmatisch äquivalent. In diesem Sinne besteht in solchen Fällen daher keine freie Variation, weshalb diese Konstruktionen ausgeschlossen wurden.
} 
gesondert behandelt, jedoch nicht gänzlich verworfen, sondern so annotiert, dass betreffende Formen integriert, isoliert oder exkludiert betrachtet werden können, was ebenso für die Vorkommen von haben und sein als Vollverben gilt.

Darüber hinaus wurde auch für alle übrigen Verbtypen bzw. Lexeme darauf geachtet, dass konkrete Instanzen im Gespräch nur gewertet wurden, wenn grundsätzliches Potential über das gesamte Variationsspektrum besteht, was, grob gesagt, für Nichtfaktizitätskontexte (cf. Diewald 1999: 185f.) gegeben scheint; auszuschließen wären dagegen etwa Formen des honorativen Konjunktivs (cf. Glauninger 2011), wobei diese im untersuchten Korpus - wohl weil sie doch auf besondere Kontexte begrenzt sind - nicht begegnen. Außerdem wurden Konjunktiv IIVorkommen in Phraseologismen und Zitaten nicht mitberücksichtigt.

Über die genannten Punkte hinaus wurde a priori auf Vorannahmen über Restriktionen, welche nicht aus bestehenden Analysen bekannt sind, verzichtet. Nachdem damit der konzeptionelle, methodische und phänomenologische Rahmen abgesteckt ist, können im Folgenden die Resultate der Untersuchung in den Blick genommen werden.

\section{$5 \quad$ Variation im urbanen Konjunktiv II-Spektrum - Befund und Diskussion}

Als zentrale Frage stellt sich jene nach den Bildungsmustern, nach deren Häufigkeiten sowie nach der Variationsbandbreite (sprich, ob sich eine hohe oder begrenzte Zahl unterschiedlicher Ausprägungsvarianten findet). Dabei gilt es, zu sondieren, welche Unterschiede sich diesbezüglich jeweils zwischen den beiden Städten wie auch zwischen urbanem Raum und Umland zeigen. Darüber hinaus ist die Rolle situativer und soziodemographischer Faktoren sowie jene morphopragmatischer und lexematischer Aspekte zu diskutieren.

\subsection{Wien und Graz}

Zunächst sei der Fokus auf die beiden Städte gelegt. Im Hinblick auf die präferierten Bildungstypen und deren relative Verteilungen sticht in den Interviewdaten zuallererst eine bemerkenswerte Parallelität zwischen Wien und Graz ins Auge, wie Abbildung 2 illustriert - insbesondere als sich dabei nicht nur generelle Tendenzen spiegeln, sondern die Werte auch in der Aufgliederung auf unterschiedliche Verbtypen stark parallel laufen. In beiden Städten werden die Konjunktiv II-Formen von haben und sein sowie auch jene der Modalverben mit weitaus überwiegender Mehrheit standardnormgemäß synthetisch gebildet, während sich periphrastische Formen mit würd-nur vereinzelt finden. Dies entspricht auch bisher konstatierten Befunden bestehender Untersuchungen und wird mit der hohen Gebrauchsfrequenz dieser Lexeme in Zusammenhang gebracht (cf. Nübling 1997: 108; Glauninger 2008: 239; Lotze/Gallmann 2009: 229). ${ }^{15}$

\footnotetext{
${ }^{15}$ Lotze/Gallmann ziehen in diesem Zusammenhang auch Rückschlüsse auf kognitive Aspekte: „Im hochfrequenten Teil des Verbwortschatzes ist [...] Kontrast unter den Konjugationsformen wichtiger als einfache Bildbarkeit, und zwar offenbar deswegen, weil sie im mentalen Lexikon fertig gespeichert werden“ (Lotze/Gallmann 2009: 229).
} 


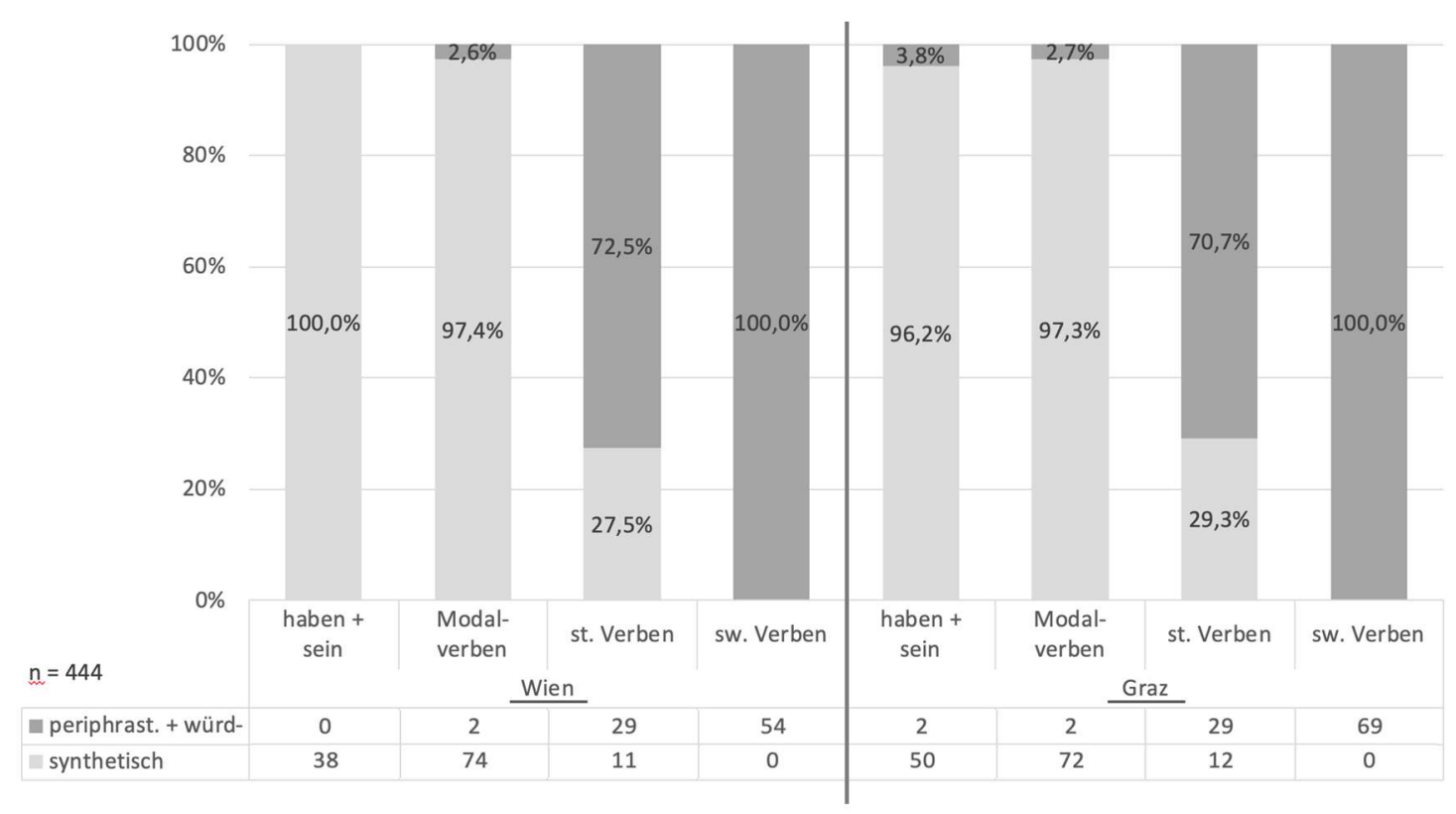

Abbildung 2: Konjunktiv II-Bildungen im urbanen Raum nach Verbtypen (Korpus A)

Bei den übrigen Verben zeigt sich dagegen umgekehrt eine klare Präferenz der periphrastischen Bildung mit würd-, was allerdings bei den schwachen Verben mit deren ausschließlicher Verwendung noch deutlicher wiegt als bei den starken Verben, welche immerhin in rund $28 \%$ (Wien, absolut 11/40) bzw. 29\% (Graz, absolut 12/41) der Fälle nach synthetischem Muster gebildet wurden. Bei den schwachen Verben spielt hier vermutlich die fehlende morphologische Differenzierung zwischen Indikativ und Konjunktiv eine bedeutende Rolle, welche zur Homophonie mit den entsprechenden Präteritumformen führt, sodass die würd-Konstruktion den Ausweg zu einer deutlichen Modus-Markierung darstellt (cf. hierzu auch Nübling 1997: 108; Thieroff 2010: 135). ${ }^{16}$

Abseits der doch bemerkenswerten Ähnlichkeit der Wiener und Grazer Daten scheinen diese Funde soweit recht unspektakulär, entsprechen sie doch den schon bekannten Tendenzen im Konjunktiv II-Spektrum und sind, wie bereits angemerkt, teils strukturell bedingt. Was aber

\footnotetext{
16 Dieser Erklärungsansatz kann freilich nur interpretativen Status beanspruchen. Einer solchen Deutung entgegengehalten werden könnte der formale Abbau des Präteritums im gesamten Oberdeutschen zugunsten einer Perfektexpansion (cf. hierzu ausführlich Fischer 2018; Pick1 2022): Denn der zunehmende Verlust der Präteritumformen und der damit einhergehende Wegfall der Homophonie hätte doch eigentlich im Verbalsystem Raum geschaffen für eine Stärkung der synthetischen Konjunktiv II-Formen (cf. hierzu auch Donhauser 1992: 227). Während diese in stärker dialektal geprägten Gebieten ohnehin Bestand haben und zudem eine größere (über die mit dem Indikativ homophonen Varianten hinausgehende) Formenvielfalt zeigen (cf. ibd.), scheinen die synthetischen Bildungen schwacher Verben in standardnäheren Domänen, wie die vorliegenden Befunde belegen, dennoch nur marginal vorhanden. Weshalb der durch den Schwund der gleichlautenden Präteritum-Bildungen „frei“ gewordene Raum im Verbalsystem nicht allgemein für die Festigung der synthetischen Konjunktiv II-Formen genutzt wurde, darüber könnte nur spekuliert werden, sodass diese Frage hier offenbleiben muss. Im Hinblick auf die nicht auftretenden -at-Konstruktionen wäre ferner der spezifisch bairische Effekt der Ablöse der Präteritalformen durch diese Konjunktive zu berücksichtigen. Schnelzer (2008: 91f.) führt hierzu unter Rückgriff auf Dal (1960) aus, inwiefern die recht starke Position des Konjunktivs sowie die Ersetzbarkeit des Präteritums durch das Perfekt zur eindeutigen Modusmarkierung geführt hat. Nun sind aber gerade diese Konstruktionen nicht in Verwendung, was noch am ehesten für die sich ausbreitende Tendenz des Ersatzes synthetischer Formen durch analytische spricht, nicht aber die Wechselwirkung mit dem Präteritumschwund anspricht.
} 
dennoch zweifellos hervorsticht, ist die geringe Ausschöpfung der Bandbreite an Varianten. Sowohl für die periphrastischen Bildungen mit tät- mit oder ohne -at-Infix als auch für die synthetischen Formen mit -at- findet sich in den Interviewdaten unter allen 28 Sprechern kein einziger Beleg. Bemerkenswert ist das insofern, als diese Varianten-Armut (bzw. dieses, wenn man so will, reduzierte Spektrum) ein stadtsprachliches Spezifikum darzustellen scheint.

Kontrastiert man die hier vorliegenden Resultate mit vergleichbaren Untersuchungen zum ruralen Raum, so zeigt sich, dass dort die gesamte Bandbreite an Konstruktionsmöglichkeiten ausgeschöpft wird, wie etwa Ziegler/Glantschnig (2013) für Osttirol zeigen, wo (unter den untersuchten Erwachsenen) keine einzige synthetisch-standardkonforme Bildung begegnet und Konstruktionen mit würd-zu nur rund 3\%, auftreten; daneben stehen analytische tat-Konstruktionen (wobei die Schreibung eine lautlich dialektale Realisierung nahelegen dürfte) mit einem Anteil von rund $26 \%$, synthetische Formen mit -at-Infix zu $63 \%$ und synthetisch-dialektale Bildungen mit Ablaut zu 8\% (cf. Ziegler/Glantschnig 2013: 298). Einen ähnlich starken Kontrast des ruralen zum urbanen Gebrauchsspektrum, wenn auch im Detail anders verteilt, zeigen, neben den bereits zitieren Studien von Bercko (2018) und Breuer/Wittibschlager (2020), insbesondere auch die Befunde zum ruralen Raum von Vergeiner/Bülow (2022) auf, wo die in der Stadt präferierten standardkonformen synthetischen Bildungen und periphrastischen würdKonstruktionen im Vergleich zu den übrigen Bildungstypen deutlich unterrepräsentiert sind (wenn man von den haben/sein-Konjunktiven absieht, welche auch hier überwiegend synthetisch realisiert sind). Die Parallelität der Daten aus Wien und Graz in Verbindung mit dem Kontrast zum ruralen Raum weist das reduzierte Variantenspektrum somit als prägendes Charakteristikum des Sprachprofils des urbanen Raums aus.

Die starke Neigung zum periphrastischen Bildungstyp kann dabei mit mehreren Faktoren abseits der bereits angesprochenen teilweisen Vermeidung von Homophonie - in Verbindung gebracht werden. Smirnova (2006: 190) etwa weist darauf hin, dass Sprecher häufig auch bei Bestehen eindeutiger und gebräuchlicher Konjunktivformen die würd-Periphrase als die „noch eindeutigere“ Form vorziehen (wobei diese größere Eindeutigkeit gleichermaßen für die tätKonstruktion gelten müsste). Zugleich deckt sich dieser Trend mit der allgemeinen Beobachtung einer Tendenz zum analytischen Sprachbau im verbalen Bereich, welche sich parallel auch im Abbau des Präteritums (zugunsten des Perfekts) zeigt (cf. hierzu etwa Fischer 2018). Dies stellt dabei kein modernes Phänomen dar, wie Auer (2007) anhand historischer Daten beschreibt:

The main reason for the würde-periphrasis lies in the Austrian language situation in the eighteenth century, with a primary dialect and a prestige variety. As the corpus data show, the forms of the past subjunctive are very different in spoken and written language. The forms used in spoken language are dissimilar to the grammatical past subjunctive forms, which are based on the synthetic past indicative forms. However, the data from dialect poetry show that Austrians seem to feel uncomfortable with synthetic past indicative forms, which was also claimed by Popowitsch (1754).

(Auer 2007: 14) 
Da die Präteritumform die Ableitungsbasis des Konjunktiv II darstellt, führe deren Fehlen im gesprochenen Deutsch folglich zu Problemen bei der Bildung der Konjunktiv II-Formen (cf. Auer 2007: 14 sowie zum Problem der Ableitbarkeit auch Nübling 1997: 107). ${ }^{17}$

Einen weiteren interessanten Erklärungsansatz liefert Stöckle, welcher die Tendenz zur analytischen Bildung mit Sprachkontakt in Verbindung bringt: Dass die periphrastischen Formen so ein Befund der Untersuchung - häufiger an den Rändern des deutschen Sprachraums vorkommen, könne möglicherweise damit in Zusammenhang stehen, dass sie regelmäßig gebildet und damit kognitiv einfacher zu verarbeiten seien (cf. Stöckle 2020). Dies ließe sich fallweise auch auf den urbanen Raum übertragen, stellt dieser doch einen Paradeschauplatz diversen Sprachkontakts dar (cf. etwa Androutsopoulos et al. 2013: 17-21; Coulmas 2017; Smakman/Heinrich 2017). Eine solche Interpretation kann einstweilen nur in den Raum gestellt werden und bedürfte freilich eingehenderer Untersuchungen, welche vor allem auch urbane Sprecher mit nicht-deutschem Muttersprachhintergrund einbeziehen müssen.

Was das Verhältnis der beiden Auxiliare betrifft, lassen sich auf jeden Fall urbane Tendenzen im Rahmen des Dialekt-Standard-Kontinuums ins Treffen führen. Die Städte sind im Allgemeinen stärker standardsprachlich geprägt und unterlaufen zudem zunehmende Standardisierungstendenzen (cf. hierzu etwa auch Glauninger 2008), weshalb die als dialektaler und regional begrenzter einzustufende tät-Variante ${ }^{18}$ (cf. Brinckmann/Bubenhofer 2012: 161; Ziegler/Glantschnig 2013: 297) zunehmend geringere Vorkommen zeigt.

Daneben besteht freilich eine gewisse Ungewissheit, was die diachrone Verteilung der beiden Auxiliare für die Konjunktivbildung in Gesprächsdaten betrifft. Für die Sprachräume Wien und Graz (inkl. ihres Umlands) lassen sich keine historischen Daten belegen, die eine dominante tät-Variante aufzeigen würden. Hierzu kann nur das dialektologische Wissen über den bairischen Sprachraum herangezogen werden, welches die dialektalen periphrastischen Konjunktivbildungen mit tun-Auxiliar beschreibt (cf. bspw. Zehetner 1978). Lenz (2016) verweist auch auf die Wenkerbögen zum Phänomen, in denen sich ein ,südlich dominierendes täte von den nördlichen Alternativkonstruktionen abgrenzt“. Ebenso nennt Schnelzer (2008: 81) für das Bairische den Konjunktiv mit der Bildung aus dem Auxiliar doa (bei ihm ,tun') als die häufigste Variante. Verhältnismäßig junge Perzeptionsdaten aus dem Atlas zur deutschen Alltagssprache (AdA) stützen diese Einsicht mit Umfrageergebnissen zum Konjunktiv II (cf. AdA: f18c, f08bc, r8-f4m-2). Demnach bestätigt sich die Darstellung des Sachverhalts über den Sprachraum hinweg, wie es Lenzhofer (2017) bspw. schon mit Schwerpunkt auf Osttirol beschreibt und auch Vergeiner/Bülow (2022) anhand ihrer basisdialektalen Daten belegen können.

\footnotetext{
${ }^{17}$ Diese Deutung setzt allerdings die Annahme voraus, dass jede Konjunktiv II-Bildung notwendigerweise auch synchron aus der jeweiligen Präteritum-Form abzuleiten ist und lässt die Möglichkeit außer Acht, dass der Konjunktiv II als etabliertes Paradigma in verselbständigter Form bestehen kann. In letzterem Fall wäre davon auszugehen, dass nicht jede Konjunktiv II-Realisierung via Präteritum-Paradigma prozessiert würde, sondern direkt als Konstruktion über ein synchron sprachstrukturell verankertes Konjunktiv-Paradigma gebildet würde (cf. hierzu auch Bittner/Köpcke 2010: 33-38, die in diesem Zusammenhang Daten aus dem Schweizerdeutschen behandeln, welche im betreffenden Kontext der Argumentation jedoch mit dem vorliegenden Datenmaterial aus dem bairischösterreichischen Raum vergleichbar scheinen).

18 Auer (2007: 15) sowie Stöckle (2020) weisen die tät-Konstruktion auch historisch als stigmatisierte Variante aus.
} 
Für die hier im Fokus stehenden konkreten Gebiete um Wien und Graz muss eine eingehendere diachrone Auslotung der Verhältnisse dagegen (einstweilen) Desiderat bleiben, da die historischen Daten für die betreffenden Ortspunkte der urbanen Peripherie auf kleinräumiger Ebene fehlen. Aus synchroner Perspektive lässt sich bezüglich des Auxiliar-Gebrauchs aber als klarer Befund für beide Großräume die starke Dominanz der würd-Konstruktion bestätigen.

\subsection{Urbaner Raum und städtisches Umland}

Hier schließt sich die Frage an, wie es sich nun mit der Relation zwischen den Städten und ihrem unmittelbaren Umland verhält - und wo das Umland seinerseits hinsichtlich der konstatierten Stadt-Land-Opposition einzuordnen ist. Die Antwort darauf fällt klar aus und sei den spezifischeren Befundausführungen gleich vorweggenommen: Die Agglomerationen (zumindest die hier erfassten) weisen in Bezug auf das Konjunktiv II-Spektrum eine starke Ähnlichkeit zum städtischen Bild auf. Setzt man etwa alle urbanen Gewährspersonen in Kontrast zu den Sprechern der sechs Agglomerationen und vergleicht in den Interviewdaten synthetische mit periphrastischen Konstruktionen (abzüglich aller Vorkommen von sein, haben und der Modalverben), so zeigen sich (siehe Abbildung 3) idente Verteilungen für beide Bereiche.

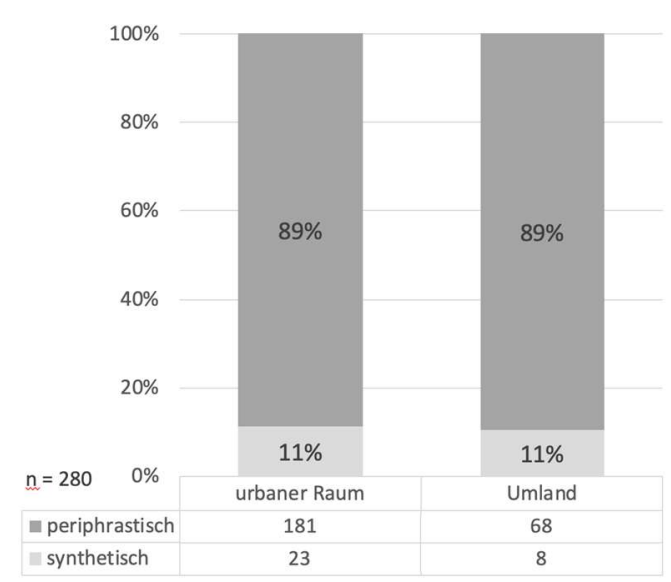

\section{Abbildung 3: Periphrastische und synthetische Konjunktiv II-Bildungen: urbaner Raum vs. städtisches} Umland (Korpus A)

Zwar weisen die Umland-Gemeinden keine derart strenge Einschränkung auf die zwei Optionen standardnorm-synthetischer und würd-periphrastischer Konstruktionen auf, sondern zeigen ein größeres Formenspektrum, doch bleiben die Zahlen anderer Bildungstypen in den Interviews dennoch gering: So findet sich in den Umland-Daten ein Vorkommen von rund 5\% (5/101 absolut) an synthetischen Formen mit -at-Infix ${ }^{19}$ sowie innerhalb des periphrastischen Musters eine Häufigkeit von rund 4\% (3/70 absolut) an Bildungen mit tät-Auxiliar ${ }^{20}$. In Bezug auf die Lautung, welche grob als zusätzliches Ausprägungsmerkmal in der Auswertung mitberücksichtigt wurde, ${ }^{21}$ stehen den rund 100\% (448/449) standardnahen Realisierungen in den

\footnotetext{
19 Signifikant nach dem exakten Test nach Fisher: $\mathrm{p}=0.0017$.

20 Signifikant nach dem exakten Test nach Fisher: $\mathrm{p}=0.0197$.

21 Dabei wurde jedem Konjunktiv II-Vorkommen im Korpus zusätzlich der Wert „standardnäher“ vs. „standardferner“ zugewiesen, wobei sich das Urteil an der stammvokalischen Realisierung orientierte (Formen mit Apokope, wie ich würd/wär etc., wurden dabei als „standardnäher“ eingestuft, sofern der Stammvokal standardnormgemäß realisiert wurde). Dieses Vorgehen kann allerdings freilich nur als annäherungsweise Repräsentation der
} 
Städten im Umland nur rund 8\% (13/171) standardferne vs. 92\% (158/171) standardnahe Realisierungen ${ }^{22}$ gegenüber.

Erweitert man das Bild um die vertikale Perspektive (formelles Interview vs. informelle Freundesgespräche), so zeigen sich dennoch gewisse Unterschiede zwischen urbanem Raum und städtischem Umland. Zwar offenbart der Vergleich von synthetischen und periphrastischen Vorkommen (unter Ausschluss von sein/haben und der Modalverben) wiederum ein eher gleichverteiltes Bild, doch finden sich innerhalb der beiden Konstruktionstypen durchaus Divergenzen. Dies betrifft zum einen den Gebrauch der Auxiliare (siehe Abbildung 4), für die

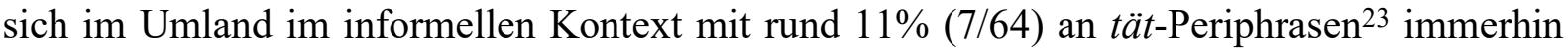
ein gewisses Vorkommen zeigt.

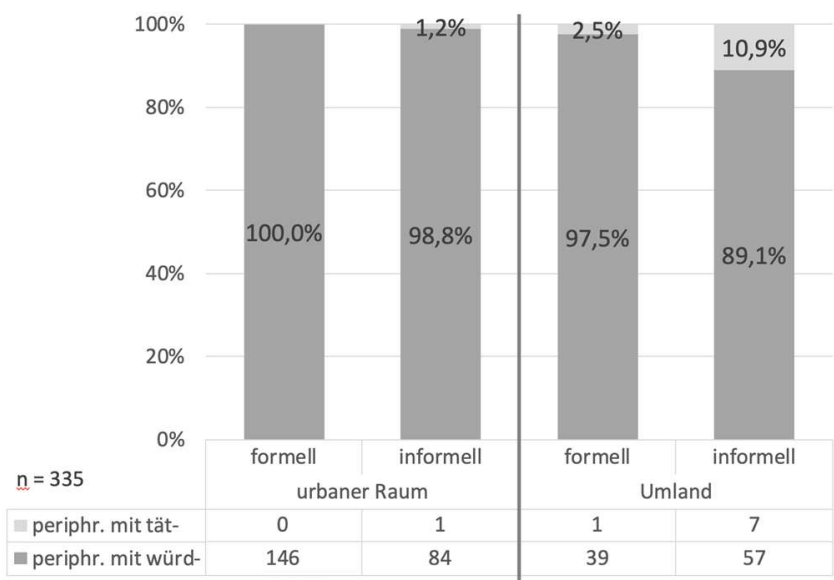

\section{Abbildung 4: Periphrastische Konjunktiv II-Bildungen mit tät- und würd-: urbaner Raum vs. städtisches Umland/formelles vs. informelles Setting (Korpus B)}

Zum anderen findet sich unter den synthetischen Formen eine nennenswerte Zahl an Instanzen mit dem Infix -at- (siehe Abbildung 5). Während im städtischen Raum im informellen Setting nur 1\% (2/198) (und im formellen Kontext kein einziges Vorkommen) an mit zusätzlichem Flexiv gebildeten Formen aufscheint, sind es im Umland im Interview rund 7\% (4/60) und im Freundesgespräch 14\% (12/87). ${ }^{24}$ Hier muss eingeräumt werden, dass die absoluten Belegzahlen nicht sehr hoch sind, was die Aussagekraft freilich etwas schmälert, doch sollten die Befunde dennoch Hinweise auf tendenzielle Muster liefern können.

lautlichen Gegebenheiten betrachtet werden und soll eher einem zusätzlichen Eindruck dienen, als dass hierbei Ansprüche auf spezifischere phonetische Auswertungsbefunde gestellt werden könnten.

22 Signifikant nach dem exakten Test nach Fisher: $\mathrm{p}<0.0001$.

23 Das Ergebnis ist hinsichtlich des Unterschieds zwischen Stadt und Umland im informellen Kontext nach dem exakten Test nach Fisher signifikant: $\mathrm{p}=0.0212$.

${ }^{24}$ Die Unterschiede zwischen Stadt und Umland sind nach dem exakten Test nach Fisher jeweils signifikant: formeller Kontext: $\mathrm{p}=0.0028$, informeller Kontext: $\mathrm{p}<0.0001$. 


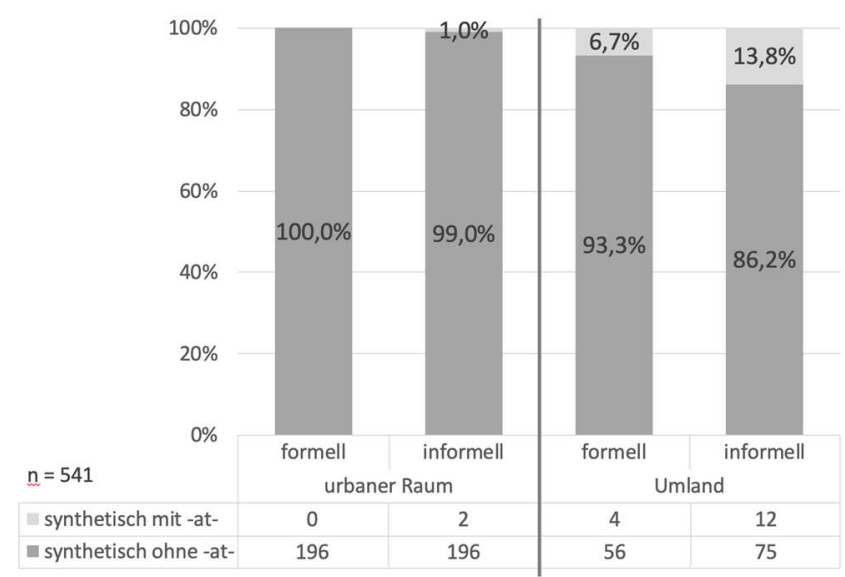

Abbildung 5: Synthetische Konjunktiv II-Bildungen mit und ohne Infix: urbaner Raum vs. städtisches Umland/formelles vs. informelles Setting (Korpus B)

Im Hinblick auf die vertikale Dynamik zeigt sich zudem sowohl für die Auxiliare als auch für das Flexiv -at-, dass der Unterschied zwischen Freundesgespräch und Interview im Umland größer ist als im urbanen Raum. Die vertikale Differenzierung erweist sich also in der Stadt als weniger ausgeprägt als in den umliegenden Gemeinden. Dies könnte entweder darauf hindeuten, dass die Sprecher der Umlandgemeinden eine höhere Sensibilität in Bezug auf den Interaktionskontext aufweisen bzw. sich stärker an unterschiedliche Situationen anpassen oder auch dass die urbanen Sprecher für die unterschiedlichen Formalitätsgrade - zumindest in diesem spezifischen grammatischen Phänomenbereich - keine Sprechlagenunterschiede auf die divergenten Situationen applizieren.

Weiters lässt sich hier das Verhältnis zwischen Städten und Agglomerationen auch hinsichtlich des Dialekt-Standard-Kontinuums sondieren. Nachdem die Konstruktionen mit tät-Auxiliar sowie die Bildungen mit -at-Infix in Relation zu den hier als Opposition gefassten Varianten als die jeweilig dialektaleren gelten, deuten die Ergebnisse darauf hin, dass das städtische Umland eine stärkere Neigung zu dialektnäheren Realisierungen als die Städte aufweist. Zugleich aber belegen die Zahlen, dass dies eine nur schwach ausgeprägte Neigung ist und dass sowohl Formenbandbreite als auch Häufigkeitsverteilungen stark an das Profil der Stadt angenähert sind. Hinsichtlich der Hintergründe bleibt hier gewiss ein Interpretationsspielraum offen, doch dürfte dieses Bild dennoch auf einen sprachlichen Einfluss der Städte auf die Agglomerationen im Sinne einer „contagious diffusion“ (cf. etwa. Taeldeman 2005: 263) hinweisen, wobei die Merkmals- oder Musterverbreitung über direkten Kontakt wellenförmig in Richtung benachbarter Orte erfolgt. Zusätzlich bestärkt wird die Annahme eines urbanen Einflusses auf das Umland dadurch, dass der rurale Raum, wie bereits geschildert, eine deutlich andere Prägung zeigt. Die (geographische) Nähe zur Stadt, aber vor allem auch die ökonomische und soziale Anbindung (durch die beruflichen, infrastrukturellen und kulturellen Gegebenheiten), die sich als Alltagsmobilität in Richtung urbaner Zentren auswirkt, spielen hier sicher eine Rolle in der Diffusion sprachlicher Gepflogenheiten und Gebrauchsmuster.

Vergleicht man darüber hinaus das Wiener mit dem Grazer Umland, scheint sich abzuzeichnen, dass die Wiener Agglomerationen tendenziell urbaner geprägt sind. Der Anteil synthetischer Formen in Relation zu periphrastischen ist im Grazer Umland fünfmal höher als in den Wiener 
Agglomerationen (rund $4 \%$ vs. $21 \%$ oder absolut $2 / 47$ vs. $6 / 29)^{25}$. Außerdem realisieren die Sprecher des Grazer Umlands die Konjunktiv II-Formen mehr als sieben Mal häufiger mit standardferner Lautung (rund $2 \%$ vs. $15 \%$ bzw. absolut $2 / 98$ vs. $11 / 73$ ) ${ }^{26}$ als die Sprecher der Wiener Umgebungsgemeinden. ${ }^{27}$ Wenn man der Interpretation folgt, dass es einen urbanen Trend zur Verwendung periphrastischer Formen gibt sowie die stärker standardsprachliche Prägung der Stadt mitbedenkt, so würden diese Resultate darauf hindeuten, dass das Wiener Umland an sich städtischer geprägt ist ${ }^{28}$ oder aber dass Wien einen größeren Einfluss auf sein Umland ausübt als Graz. Dies wäre im Sinne einer Diffusionsdynamik, wie sie dem Konzept des ,gravity models“" entspricht, zu interpretieren, welches von der unterschiedlichen Wirkkraft verschiedener urbaner Zentren ausgeht und als Erklärungsmodell Verwendung findet, ,in order to weigh the socioeconomic strength of cities and towns and, hence, their strength to transmit linguistic elements to other (smaller) centres“ (Taeldeman 2005: 277), wobei Taeldeman zusätzlich den Stellenwert sozial-psychologischer Faktoren wie etwa ,the attitudinal relation between the urban centre and its hinterland“" (Taeldeman 2005: 283) betont.

Geht man von einem solchen größeren Gewicht Wiens aus (welches sich nicht nur aus der flächen- und einwohnerzahlbezüglichen Größe, sondern auch dem Status als Hauptstadt sowie bedeutendstem Medienstandort des Landes ergibt), so lässt sich nun im Hinblick auf die sprachliche Einflusskraft eine Brücke zum obigen Städte-Vergleich schlagen. Der unter 5.1 dargestellte Befund zur Parallelität der Ergebnisse der beiden Städte in Kombination mit den Resultaten aus den Agglomerationen könnte darauf hindeuten, dass sich die Konjunktiv II-Gebrauchsmuster in einem mehrstufigen Prozess verbreiten: Die Diffusion könnte demnach zunächst in Form einer ,hierarchical diffusion“ (cf. Taeldeman 2005) erfolgen, also sprunghaft ohne die Bedingung direkter Nachbarschaft vom größeren urbanen Zentrum Wien auf das in Relation kleinere (und weniger ,gewichtige“) Graz und von dort - so die Annahme - weiter auf wiederum kleinere Städte, woraufhin in Folge bzw. teils zugleich zudem eine weitere Verbreitung - in Form einer „contagious diffusion“ - von den Städten auf die Umlandgemeinden und von dort auf deren Nachbarorte erfolgt. ${ }^{29}$ Diese Überlegungen haben freilich nur interpretativen Status, sollen den dargestellten Daten aber dennoch als mögliche Ausdeutung an die Seite gestellt werden.

\footnotetext{
${ }^{25}$ Signifikant nach dem exakten Test nach Fisher: $\mathrm{p}=0.0480$.

${ }^{26}$ Signifikant nach dem exakten Test nach Fisher: $\mathrm{p}=0.0022$.

${ }^{27}$ Ein zusätzlicher Kontrast hinsichtlich der Konstruktionen mit Infix, welcher nahelegt, dass die Sprecher des Grazer Umlands viermal häufiger die dialektnäheren Formen mit -at- verwenden als die Gewährspersonen der Wiener Agglomerationen (rund $2 \%$ vs. $8 \%$ bzw. absolut $1 / 52$ vs. $4 / 49$ ), erweist sich als statistisch nicht signifikant $(\mathrm{p}=0.1960$ nach dem exakten Test nach Fisher). Ein solcher Unterschied kann auf Basis der verfügbaren Daten daher nicht statistisch bestätigt werden.

28 Das würde auch damit übereinstimmen, dass die Grazer Agglomerationen im Vergleich zum Wiener Umland einen etwas ruraleren Charakter in Bezug auf Faktoren wie Infrastruktur, Einwohnerzahl, Kultur- und Freizeitangebote etc. aufweisen.

${ }^{29}$ Zugleich ist für Wien wohl auch ein unmittelbarer Einfluss auf ganz Österreich anzunehmen, da von der Hauptstadt ausgehend vor allem durch ihre Mediendominanz Diffusionsbewegungen zu erwarten sind, die weder über die Peripherie, noch über andere Zentren verlaufen, sondern direkt alle Sprecher im Land erreichen. Ein Abwägen unterschiedlicher Wirkkräfte in diesem Sinne kann hier allerdings freilich nicht geleistet werden, sind diese Mechanismen letztlich aus den Daten in keiner Weise ablesbar.
} 


\title{
5.3 Die Faktoren Geschlecht und Alter
}

Nimmt man zusätzlich soziodemographische Parameter in den Blick, zeigen sich auch hier nennenswerte Tendenzen hinsichtlich der Konjunktiv II-Ausprägungen. In Bezug auf geschlechterspezifische Verwendungsweisen stellt sich in Betrachtung der Interviewdaten heraus, dass es bei der Verwendung synthetischer gegenüber periphrastischen Konstruktionen deutliche Unterschiede gibt: Während männliche Sprecher rund 17\% (absolut 21/123) der Formen synthetisch realisieren, sind es bei den Sprecherinnen nur 6\% (10/157) ${ }^{30}$ (siehe Abbildung 6).

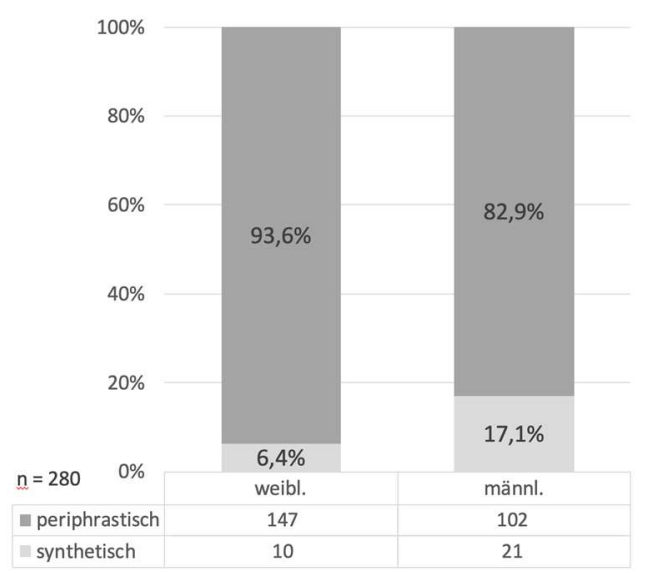

\begin{abstract}
Abbildung 6: Periphrastische und synthetische Konjunktiv II-Bildungen: geschlechterspezifische Variation (Korpus A)
\end{abstract}

Dies könnte damit in Zusammenhang gesehen werden, dass, wie häufig konstatiert (cf. etwa Labov 2001), Sprecherinnen eine treibende Kraft bei der Diffusion innovativerer Formen oder Merkmale darstellen. Insofern als die synthetischen Konjunktiv II-Varianten als konservativer begriffen werden können und die Sprecherinnen diese eher abgelegt haben, scheinen sich die männlichen Sprecher im Vergleich stärker dem weniger „modernen“ Bildungstyp verbunden zu erweisen.

In Bezug auf die beiden Altersgruppen zeigen sich in den Interviewdaten dagegen nur geringe Differenzen: Während die älteren Sprecher rund 13\% (9/68) der Konstruktionen nach synthetischem Bildungsmuster produzieren, sind es bei den jüngeren mit 10\% (22/212) zwar weniger, der Unterschied ist jedoch gering und kann bei bestehender Datenlage auch nicht als statistisch signifikant belegt werden. ${ }^{31}$ Dies ändert sich allerdings im informellen Gesprächskontext. Fokussiert man auf die Daten aus den Freundesgesprächen, erweist sich der Faktor Alter als deutlich prägend (siehe Abbildung 7).

\footnotetext{
30 Signifikant nach dem exakten Test nach Fisher: $\mathrm{p}=0.0066$.

${ }^{31}$ Nach dem exakten Test nach Fisher: $p=0.5099$.
} 

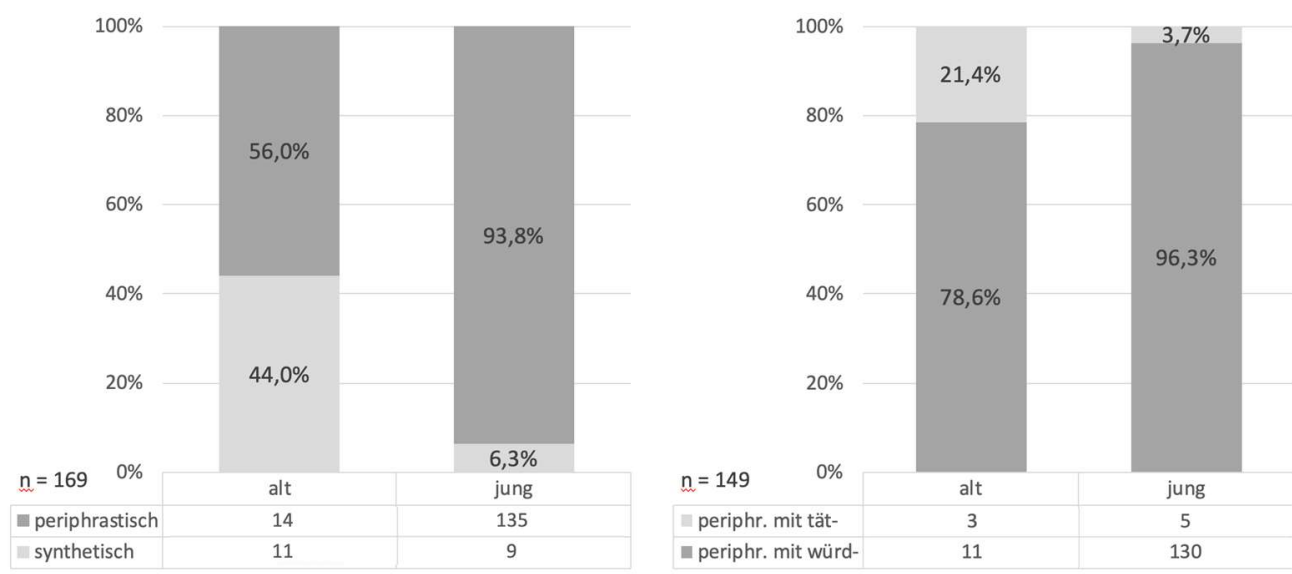

Abbildung 7: Periphrastische und synthetische Konjunktiv II-Bildungen sowie periphrastische Konstruktionen mit tät- und würd-: altersspezifische Variation (Korpus B)

Betrachtet man die Verteilung der Konstruktionstypen, so zeigt sich, dass die ältere Sprechergruppe zu rund $44 \%$ synthetische Formen (gegenüber 56\% periphrastischen bzw. absolut 11/25 vs. 14/25) verwendet; demgegenüber stehen nur rund $6 \%$ synthetische Bildungen (9/144) in der jüngeren Gruppe. ${ }^{32}$ Innerhalb des periphrastischen Typs wiederum werden von den älteren Sprechern rund 21\% (3/14) der Formen mit tät-Auxiliar gebildet, während diese Variante unter den jüngeren Gewährspersonen zu nur rund 4\% (5/135) vertreten ist. ${ }^{33}$ Wenn die absoluten Werte hier teils auch nur geringe Zahlen liefern können, so sollen diese Befunde zumindest dennoch Hinweise auf tendenzielle Trends bieten können. Die häufigere Verwendung der periphrastischen Formen sowie in deren Rahmen der würd-Konstruktion bei jungen Sprechern deckt sich zudem mit den Resultaten bei Breuer/Wittibschlager (2020: 159). Dabei vermag der Altersgruppenvergleich zudem diachrone Entwicklungen zu simulieren, sodass die hier aufscheinenden Relationen im Sinne eines apparent-time-Vergleichs als Hinweise auf Sprachwandeltendenzen, nämlich in Richtung von über die Zeit steigenden Verwendungsfrequenzen von periphrastischen Bildungen sowie von jenen mit würd-Auxiliar, gedeutet werden können, was die Fortsetzung der diachron schon belegten Entwicklungen (wie unter 5.1 diskutiert) bestätigt. Dass sowohl das synthetische Bildungsmuster (cf. z. B. Donhauser 1992: 227) als auch das Auxiliar tät- als die jeweils vergleichsweise konservativeren Varianten eingestuft werden (cf. hierzu auch die diachron zurückliegenden Befunde bei Stöckle 2020), wobei Letzteres zumal eine standardfernere Markierung trägt (cf. etwa Ziegler/Glantschnig 2013: 297; Lenzhofer 2017: 282), stützt diese Interpretation. Ebenso weist der Befund Glauningers zum jugendlichen Variantenspektrum in Wien in diese Richtung:

\footnotetext{
32 Signifikant nach dem exakten Test nach Fisher: $\mathrm{p}=<0.0001$.

${ }^{33}$ Signifikant nach dem exakten Test nach Fisher: $\mathrm{p}=0.0282$.
} 
Da für eine immer größer werdende Zahl an Wiener Jugendlichen die (intendierte) Standardvarietät des Deutschen endgültig als ,unmarkierte“ Varietät innerhalb einer immer größeren Menge alltäglicher Kommunikationssituationen fungieren dürfte, hat folgerichtig auch die im standardsprachlichen Bereich des Gesamtdeutschen längst verbreitetste (weil polyfunktionale) Konjunktiv-Form würde + Inf. in Wien innerhalb eines relativ kurzen, „erlebbaren“ Zeitabschnitts das Potenzial dazu entwickelt, auch sprechsprachlich als varietätenspezifisch „unmarkierte“ - und somit universell einsetzbare Konjunktiv-(,Einheits-“)Form zu fungieren.

(Glauninger 2008: 241)

\section{4 Überlegungen zu morphopragmatischen und lexematischen Faktoren}

Zuletzt ist an das untersuchte sprachliche Material auch noch die Frage zu stellen, ob es auch abseits der lokalen und sozialen Parameter Aspekte gibt, welche Einfluss auf die Realisierung des Konjunktiv II haben. Dies betrifft etwa den pragmatischen Bereich, in dessen Sphäre beispielsweise die schon erwähnten - hier ausgeschlossenen - Vorkommen mit honorativer Funktion fielen. Auch wenn die Daten hier kein klares Bild ergeben, sei auf gewisse Auffälligkeiten hingewiesen, da dennoch einige Fälle von anscheinend verfestigten Strukturen vorkommen, welche zumindest eine Neigung zu bestimmten Bildungstypen zeigen und ein geringeres Variationspotential aufzuweisen scheinen. Hierzu zählen Fügungen der Form würd(e) ich sagen/tät ich sagen, welche in der Funktion von Disfluenzmarkern bzw. Vagheitsindikatoren (cf. Imo 2007: 123; Stein 1995: 242) auftreten, wie sich im folgenden Beispiel ${ }^{34}$ zeigt:

(1) (-) ower nur nur is er grod in dem bereich is er (-) is er (-) Üwerkandidelt; weil_a (-) OIS personalisiert; =net, (---) er er- (1.7) es wird OIS an an irgendwöchen personen aufghängt, (--) und und INholtlich is er (---) ouft amoi recht dürr:; würde ich- (--) würde ICH sogen; ober oKAY des is hoit a- (---) gaunz gaunz (-) perSÖNliche meinung; (ULA-Korpus, 4017_THAL_alt_m_CAF, Seg. 220-231)

Der Sprecher übt hier Kritik am Dorfpfarrer, markiert diese am Ende aber relativierend - und den assertiven Gehalt der Äußerung rückstufend (Imo 2007: 123) - als subjektiv: nicht nur mit dem dann folgenden expliziten Verweis auf die persönliche Meinung, sondern schon zuvor mit der Fügung würde ICH sogen. In diesem Funktionsrahmen treten diese entsprechenden Konstruktionen vorrangig in der periphrastischen Bildung (und zumeist mit würd-) auf. Ähnliches, aber umgekehrt, gilt für wüsst ich nicht/wüsst $i$ net, welches wiederum tendenziell eher synthetisch (fallweise auch mit -at-Flexiv) realisiert ist und ebenso ein geschmälertes Variationspotential aufzuweisen scheint.

Dennoch ist es auf Basis der bestehenden Datenlage nicht möglich, für diese Fügungen Allgemeinaussagen in Bezug auf Bildungsrestriktionen zu treffen, da das in Betracht genommene Material für solcherlei Phrasen, welche im Korpus nur vereinzelt-verstreut unterkommen, einstweilen nur einen exemplarisch-explorativen Befund erlaubt. Dass hier Vorsicht im Hinblick auf voreiliges Konstatieren von Mustern geboten ist, illustriert zudem der Fall von würde gern $X$ können/tät gern X können im Sinne eines Wunsches, über eine bestimmte Fähigkeit zu verfügen. Es handelt sich um eine Konstruktion, die gewöhnlich periphrastisch begegnet, die sich im

\footnotetext{
${ }^{34}$ Die Transkription folgt den GAT 2-Richtlinien - cf. Selting et al. (2009) - mit leicht adaptierten Konventionen.
} 
Korpus jedoch sehr wohl auch in einer synthetischen Bildung findet, wie Beispiel 2 zeigt, in dem ein Sprecher sich zu Dialekten/Regiolekten äußert:

(2) (1.5) mir gfollen VIEle gut; kärntnerisch gfallt ma SEHR gut, (-) kann i KAUM imitieren, =KÖNNT i gern, (ULA-Korpus, 4057_GERAS_jung_m_AI, Seg. 626)

In diesem Sinne lassen sich schwer allgemeinere Bildungsrestriktionen formulieren. Schließlich kann nicht erschlossen werden, ob Alternativvarianten, die unmöglich oder unwahrscheinlich erschienen, möglicherweise nicht nur zufällig nicht im Korpus begegnen. Aus diesem Grund wurden bei den Erhebungen - über das Ausschließen der Formen von sein, haben und der Modalverben hinaus - auch keinerlei weitere Einschränkungen für die Wertung von Belegen vorgenommen, auch wenn sich für manche Fügungen Muster abzuzeichnen scheinen.

Dies gilt nicht nur für den morphopragmatischen Bereich, sondern ist auch auf lexematischer Ebene festzustellen. Interessant ist in diesem Zusammenhang ein Blick auf die synthetischen Belege der Untersuchung: Nimmt man wieder sein, haben sowie die Modalverben aus, so realisieren sich die verbleibenden 51 Vorkommen synthetischer Konjunktiv II-Bildungen in einer begrenzten Menge von nur 10 Lexemen, während sich die - unter dem gleichen Ausschluss gezählten - insgesamt 398 Instanzen periphrastischer Konjunktiv II-Konstruktionen auf 159 unterschiedliche Verblexeme verteilen. Betrachtet man diese Gruppe synthetisch auftretender Lexeme genauer, findet sich zudem eine interessante Verteilung, wie Tabelle 3 zeigt.

\begin{tabular}{|l|c|c|}
\hline & synthetische Vorkommen & periphrastische Vorkommen \\
\hline wissen & 31 & 3 \\
\hline brauchen & 6 & 0 \\
\hline finden & 4 & 6 \\
\hline geben & 4 & 6 \\
\hline tun $^{35}$ & 2 & 3 \\
\hline abgehen & 1 & 2 \\
\hline ankommen & 1 & 0 \\
\hline auffallen & 1 & 2 \\
\hline bringen & 1 & 3 \\
\hline passen & 1 & 5 \\
\hline
\end{tabular}

Tabelle 3: In synthetischer Realisierung auftretende Lexeme mit Vergleichswerten zu periphrastischen Vorkommen (Gesamtkorpus)

Die Aufstellung offenbart auf den ersten Blick das starke Gewicht von wissen unter den synthetischen Belegen, zumal selbiges auch etwa zehnmal häufiger synthetisch als periphrastisch realisiert wird, sodass sich für wissen eine Tendenz zum synthetischen Konstruktionstyp abzeichnet. Bei den übrigen Lexemen lässt die geringe Häufigkeit keinen allgemeineren Schluss zu. Auffällig sind allenfalls noch die Werte von brauchen, welches zweitgereiht ist und keine einzige periphrastische Realisierung aufweist; aufgrund der kleinen Zahl an Vorkommen kann dieser Kontrast aber auch dem Zufall geschuldet sein. Zusätzlich sei hier angemerkt, dass abzüglich der Modalverben sowie sein, haben, wissen und brauchen kein Sprecher mehr als zwei

35 Hierbei handelt es sich um Instanzen von tun als Vollverb. 
synthetische Konjunktivkonstruktionen verwendet. Abseits der ohnehin häufig synthetisch realisierten Lexeme treten die synthetischen Formen also über das Sample verteilt auf und es finden sich keinerlei idiolektale Häufungen.

Zum Status des Lexems wissen werden sicher Folgeuntersuchungen notwendig sein, die auf breiterer Datenbasis klarer machen, ob es für künftige Analysen eventuell an die Seite von sein, haben sowie der Modalverben gestellt werden und ebenso für allgemeine Häufigkeitserhebungen isoliert behandelt bzw. ausgenommen werden müsste. Die hier diskutierten lexematischen und auch morphopragmatischen Aspekte können einstweilen nur als kurz belichtete Fragmente gelten, die zusätzliche für das Konjunktiv II-Spektrum relevante Aspekte darstellen könnten, jedoch noch einer systematischen Bearbeitung bedürfen, um einen begründeten Befund ableiten zu können - insbesondere auch zu ihrer stadtsprachlichen Spezifik.

\section{$6 \quad$ Zusammenfassung}

Der Konjunktiv II an sich ist freilich kein stadtspezifisches Phänomen; auch finden sich keine einzelnen besonders für den urbanen Raum charakteristischen Bildungsformen. Sehr wohl aber zeigen sich umgekehrt einerseits ein Fehlen bestimmter Varianten sowie andererseits spezifische Häufigkeiten und Verteilungsmuster, welche sich als kennzeichnend für den Sprachgebrauch in der Stadt erweisen. In diesem Sinne können die Befunde aus den Analysen des Konjunktiv II-Spektrums als Hinweise dafür gelten, dass die Stadt als sozial definierter Raum mit ihren besonderen soziopragmatischen Bedingungen durchaus ein eigenes sprachliches Profil aufweist.

Dies wird zum einen dadurch belegt, dass die Verteilungsmuster der Konjunktiv II-Formen in den beiden Städten eine auffällige Parallelität zeigen und sich die jeweiligen Häufigkeiten in Wien und Graz kaum unterscheiden. Zusätzlich weist der urbane Raum im Kontrast zu ruralen Bereichen ein stark reduziertes Formenspektrum auf, welches auf zwei Konstruktionstypen beschränkt ist: den synthetisch-standardnormgemäßen sowie den periphrastischen mit würd-Auxiliar. In Bezug auf deren relative Häufigkeiten findet sich, abseits von sein/haben sowie der Modalverben, welche in überwiegender Mehrheit synthetisch gebildet werden, eine klare Präferenz zur periphrastischen Bildung mit würd-. Dieser Befund harmoniert mit einer allgemeinen Tendenz zum analytischen Sprachbau im verbalen Bereich sowie mit - insbesondere für den urbanen Raum angenommenen - Standardisierungstendenzen des Deutschen in Österreich. Zudem könnte die urbane Mehrsprachigkeit eine Rolle für die vermehrte Verwendung der in ihrer Bildung transparenteren periphrastischen Formen spielen - eine Interpretation, die allerdings noch tiefergehender Untersuchungen bedarf.

Im Hinblick auf das Verhältnis zwischen dem urbanen Raum und dessen Umland können insbesondere für formelle Kontexte nur geringe Unterschiede festgestellt werden. Dies ändert sich allerdings unter einer vertikalen Perspektivierung: In informellen Situationen werden von Sprechern der Agglomerationen im Gegensatz zur Stadt durchaus auch Bildungen mit -at-Infix sowie Konstruktionen mit tät-Auxiliar gebraucht, sodass für deren Repertoire ein breiteres Konjunktiv II-Formenspektrum nachgewiesen werden kann. Bemerkenswert hinsichtlich des StadtUmland-Vergleichs sind vor allem zwei Befunde: Zum einen zeigt sich im urbanen Raum eine deutlich geringere vertikale Differenzierung. Während sich dort nur marginale Unterschiede zwischen informellen und formellen Settings finden, ist die Variantenwahl der Sprecher des 
Umlands stärker situationsspezifisch geprägt. Zum anderen weisen die Agglomerationen eine etwas größere Neigung zu dialektnäheren Realisierungen auf, zeigen jedoch zugleich im Kontrast zum ruralen Raum eine deutliche Nähe zu den Gebrauchsmustern der Stadt, was auf den sprachlichen Einfluss der urbanen Zentren auf ihr Umland hinweist.

Nimmt man soziodemographische Faktoren in den Blick, so finden sich sowohl geschlechterals auch altersspezifische Differenzen. So verwenden Frauen im Vergleich deutlich weniger synthetische Formen als Männer und neigen damit stärker zum „,moderneren“ Bildungstyp. In Bezug auf den Altersvergleich sind es die älteren Sprecher, welche stärker zu den vergleichsweise „konservativeren“ Varianten tendieren. Insbesondere in den informellen Settings verwenden sie deutlich mehr synthetische Formen als die jüngere Gruppe und innerhalb des periphrastischen Konstruktionstyps weitaus häufiger tät- als Auxiliar. Diese Resultate fundieren die Annahme von - sich schon historisch abzeichnenden und sich nunmehr fortsetzenden Sprachwandeltendenzen hin zu analytischen Bildungen sowie zum vermehrten Gebrauch von würd-als Auxiliar.

Die funktionalen und verbspezifischen Aspekte im Zusammenhang mit dem Konjunktiv IISpektrum weisen dagegen keine eindeutigen Tendenzen aus. Zwar scheinen gewisse Fügungen in spezifischen diskurspragmatischen Funktionen, wie würd(e) ich sagen/tät ich sagen, bestimmte Bildungstypen zu bevorzugen, doch müssen weitere Untersuchungen eine größere Menge je vergleichbarer Vorkommen in den Blick nehmen, um einen systematischen Befund leisten zu können. Selbiges gilt auch für die Zahlen zu lexematischen Auffälligkeiten: Zwar kann eine beachtliche Einschränkung des synthetischen Bildungstyps auf nur zehn Lexeme (zusätzlich zu den ohnehin vorrangig synthetisch gebildeten Formen von sein und haben und den Modalverben) konstatiert werden, doch lassen sich bei bestehender Datenbasis noch keine allgemeinen Aussagen ableiten. Dieser Bereich bedarf insbesondere auch zur Frage einer diesbezüglichen stadtsprachlichen Spezifik noch weiterführender Analysen.

Während damit also noch einige Fragen offenbleiben, können dem dargelegten Befund dennoch essenzielle Erkenntnisse entnommen werden. Zentral sind hierbei die offenbar spezifisch urbanen Muster, welche sich in den Verteilungen der Konjunktiv II-Varianten abgebildet finden: Die gravierende Ähnlichkeit der Daten aus Wien und Graz in Kombination mit dem Kontrast zum Spektrum und den Häufigkeiten des ruralen Raums weisen darauf hin, dass das konstatierte Konjunktiv II-Profil einen Teil der sprachlichen Charakteristik der Stadt repräsentiert.

\section{Literaturverzeichnis}

AdA = Elspaß, Stephan/Möller, Robert (2003-): Atlas zur deutschen Alltagssprache (AdA). Open-Access-Publikation. atlas-alltagssprache.de/ [27.05.2021].

Androutsopoulos, Jannis et al. (2013): „Facetten gesellschaftlicher Mehrsprachigkeit in der Stadt“. In: Redder, Angelika et al. (eds.): Mehrsprachige Kommunikation in der Stadt. Das Beispiel Hamburg. Münster etc., Waxmann: 13-27. (= Mehrsprachigkeit 37).

Auer, Anita (2007): „,Österreichisches Deutsch ist eine würde-volle Sprache‘. The Subjunctive Mood in Eighteenth-Century Austria“. Leiden Papers in Linguistics 4/1: 1-20.

Auer, Peter (2005): „Europe's Sociolinguistic Unity, or: A Typology of European Dialect/Standard Constellations“. In: Delbecque, Nicole/Auwera, Johan van der/Geeraerts, Dirk (eds.): Perspectives on Variation. Berlin, de Gruyter Mouton: 7-42. (= Trends in Linguistics.

ISSN 1615-3014 
163).

Bellmann, Günter (1983): „Probleme des Substandards im Deutschen“. In: Mattheier, Klaus J. (ed.): Aspekte der Dialekttheorie. Tübingen, Niemeyer: 105-130.

Bercko, Nina (2018): „Südmittelbairische Verbalflexion in alltagssprachlicher Verwendung im Stadt-Land-Vergleich“. In: Adamzik, Kirsten/Maselko, Mateusz (eds.): VARIATIONslinguistik trifft TEXTlinguistik. Tübingen, Narr: 83-104. (= Europäische Studien zur Textlinguistik).

Bittner, Andreas/Köpcke, Klaus-Michael (2010): „Ich würde, wenn ich wüsste, dass ich könnte... - Der Konjunktiv zwischen Synthese und Analyse“. In: Bittner, Dagmar/Gaeta, Livio (eds.): Kodierungstechniken im Wandel: Das Zusammenspiel von Analytik und Synthese im Gegenwartsdeutschen. Berlin/New York, de Gruyter: 23-46. (= Linguistik - Impulse \& Tendenzen 34).

Breuer, Ludwig Maximilian/Wittibschlager, Anja (2020): „The variation of the subjunctive II in Austria. Evidence from urban and rural analyses“. Linguistic Variation 20/1: 136-171.

Breuer, Ludwig Maximilian (in Vorbereitung): Syntaktische Variation der modernen Regionalsprache in Wien. Dissertation, Universität Wien.

Brinckmann, Caren/Bubenhofer, Noah (2012): „,Sagen kann man's schon, nur schreiben tut man's selten“. Die tun-Periphrase“. In: Schneider, Roman/Konopka, Marek (eds.): Grammatische Stolpersteine digital. Festschrift für Bruno Strecker zum 65. Geburtstag. Mannheim, Institut für deutsche Sprache: 159-165.

Coulmas, Florian (2017): „Urbanisation and linguistic multitude“. In: Smakman, Dick/Heinrich, Patrick (eds.): Urban Sociolinguistics. The City as a Linguistic Process and Experience. London/New York, Routledge: 12-24.

Dal, Ingrid (1960): „Zur Frage des süddeutschen Präteritumsschwunds“. In: Indogermanica. Festschrift für Wolfgang Krause. Heidelberg, Winter: 1-7.

Denison, Norman (1971): „Some observations on language variety and plurilingualism“. In: Ardener, Edwin (ed.): Social Anthropology and Language. London/New York, Tavistock Publications: 157-183.

Diewald, Gabriele (1999): Die Modalverben im Deutschen. Grammatikalisierung und Polyfunktionalität. Tübingen: Niemeyer. (= Reihe Germanistische Linguistik 208).

Donhauser, Karin (1992): „Der Konjunktiv im Bairischen“. In: Weiss, Andreas (ed.): Dialekte im Wandel. Referate der 4. Tagung zur bayerisch-österreichischen Dialektologie, Salzburg, 5. bis 7. Okt. 1989. Göppingen, Kümmerle: 226-242.

Dressler, Wolfgang U./Wodak, Ruth (1982): „Sociophonetical methods in the study of sociolinguistic variation in Viennese German“. Language in Society 11/3, 339-370.

Duden (2016): Die Grammatik. Unentbehrlich für richtiges Deutsch. 9., vollständig überarbeitete und aktualisierte Auflage. Berlin: Dudenverlag.

Eisenberg, Peter (2005): „Das Verb als Wortkategorie des Deutschen. Zum Verhältnis von synthetischen und analytischen Formen“. In: Schaeder, Burkhard/Knobloch, Clemens (eds.): Wortarten und Grammatikalisierung. Perspektiven in System und Erwerb. Berlin/NewYork, de Gruyter: 21-41. (= Linguistik. Impulse \& Tendenzen 12).

Eisenberg, Peter (2006): Der Satz. Grundriss der deutschen Grammatik. 3. Auflage. Stuttgart/Weimar: Metzler. 
Ender, Andrea/Kaiser, Irmtraud (2009): „Zum Stellenwert von Dialekt und Standard im österreichischen und Schweizer Alltag - Ergebnisse einer Umfrage“. Zeitschrift für Germanistische Linguistik 37/2: 266-295.

Fabricius-Hansen, Cathrine (2000): „Die Geheimnisse der deutschen würde-Konstruktion“. In: Thieroff, Rolf et al. (eds.): Deutsche Grammatik in Theorie und Praxis. Tübingen: Niemeyer.

Fischer, Hanna (2018): Präteritumschwund im Deutschen. Dokumentation und Erklärung eines Verdrängungsprozesses. Berlin/Boston: de Gruyter Mouton (= Studia Linguistica Germanica 132).

Fishman, Joshua A. (1965): „Who speaks what language to whom and when?“. La Linguistique 1/2: 67-88.

Gal, Susan (1987): „Linguistic repertoire“. In: Ammon, Ulrich/Dittmar, Norbert/Mattheier, Klaus J. (eds.): Sociolinguistics. An International Handbook of the Science of Language and Society. Berlin/New York, de Gruyter: 286-292. (= HSK 3.1).

Gallmann, Peter (2007): „Morphologische Probleme der deutschen Konjunktive“. gallmann.uni-jena.de/Konjunktiv.pdf [27.05.2021], 1-26. [Gedruckte Fassung in: Gallmann, Peter/Lehmann, Christian/Lühr, Rosemarie (eds.): Sprachliche Motivation. Zur Interdependenz von Inhalt und Ausdruck. Tübingen, Narr: 45-80. (= Tübinger Beiträge zur Linguistik 502).

Glauninger, Manfred (2005): Form und Funktion der -(er)l-Deminutive. Am Beispiel des Grazer Deutsch. Frankfurt a. M. etc.: Lang. (= Schriften zur deutschen Sprache in Österreich 34).

Glauninger, Manfred (2008): „Synthetische und analytische ,Konjunktiv 2'-Formen im Wiener Nonstandard-Deutsch“. In: Patocka, Franz/Seiler, Guido (eds.): Dialektale Morphologie, dialektale Syntax. Beiträge zum 2. Kongress der Internationalen Gesellschaft für Dialektologie des Deutschen, Wien, 20.-23. September 2006. Wien, Praesens: 233-247.

Glauninger, Manfred (2010): „Zwischen Hochdeutsch, Dialekt und Denglisch. ,Innere Mehrsprachigkeit' und urbane Kommunikation am Beispiel der Jugendlichen im Ballungsraum Wien. In: Gaisbauer, Stephan/Petermayr, Klaus (eds.): Übergang. Kommunikation in der Stadt und an ihren Rändern. Linz, Adalbert-Stifter-Institut des Landes Oberösterreich: 181193. (= Schriften zur Literatur und Sprache in Oberösterreich 14).

Glauninger, Manfred (2011): „Zur ,(Morpho-)Pragmatik‘ von ,Konjunktiv 2'-Formen im Wiener Nonstandard-Deutsch. In: Pohl, Heinz Dieter (ed.): Klagenfurter Beiträge zur Sprachwissenschaft 34-36: 98-107.

Graf, Rainer (1977): Der Konjunktiv in gesprochener Sprache. Form, Vorkommen und Funktion untersucht an Tonbandaufnahmen aus Baden-Württemberg, Bayrisch-Schwaben, Vorarlberg und Liechtenstein. Tübingen: Niemeyer.

grammis: „Konjunktiv II“. In: Institut für Deutsche Sprache: Wissenschaftiche Terminologie. Grammatisches Informationssystem grammis. grammis.ids-mannheim.de/terminologie/401 [27.05.2021].

Hannemann, Christine (2019): „Stadtsoziologie - eine disziplinäre Positionierung zum Sozialraum“. In: Kessl, Fabian/Reutlinger, Christian (eds.): Handbuch Sozialraum. 2. Aufl. Wiesbaden, Springer: 45-68. (= Sozialraumforschung und Sozialraumarbeit 14). 
Hartung, Wolfdietrich (1981): „Sprachvariation und ihre linguistische Widerspiegelung“. In: Hartung, Wolfdietrich/Schönfeld, Helmut (eds.): Kommunikation und Sprachvariation. Berlin, Akademie-Verlag: 73-105.

Hutterer, Claus Jürgen (1978): „Der Stadtdialekt von Graz in Vergangenheit und Zukunft“. In: Steinböck, Wilhelm (ed.): 850 Jahre Graz. 1128-1978. Festschrift im Auftrag der Stadt Graz. Graz/Wien/Köln, Styria: 323-354.

Imo, Wolfgang (2007): Construction Grammar und Gesprochene-Sprache-Forschung. Konstruktionen mit zehn matrixsatzfähigen Verben im gesprochenen Deutsch. Tübingen: Niemeyer. (= Reihe Germanistische Linguistik 275).

Kaiser, Irmtraud/Ender, Andrea (2015): „Das Spektrum der Sprachvariation im alemannischsprachigen Vorarlberg und im übrigen Österreich: Realisierungen und Kategorisierungen“. In: Lenz, Alexandra N./Ahlers, Timo/Glauninger, Manfred M. (eds.): Dimensionen des Deutschen in Österreich. Variation und Varietäten im sozialen Kontext. Frankfurt a. M., Lang: 11-29. (= Schriften zur deutschen Sprache in Österreich 42).

Kerswill, Paul (2003): „Dialect levelling and geographical diffusion in British English“. In: Britain, David/Cheshire, Jenny (eds.): Social Dialectology. In honour of Peter Trudgill. Amsterdam, Benjamins: 223-243.

Kerswill, Paul/Williams, Ann (2002): ,,Salience“ as an explanatory factor in language change: evidence from dialect levelling in urban England“. In: Jones, Mari C./Esch, Edith (eds.): Language Change. The Interplay of Internal, External and Extra-Linguistic Factors. Berlin/New York, de Gruyter Mouton: 81-110. (= Contributions to the Sociology of Language 86).

Krefeld, Thomas (2015): „Sprachliche Variation im kommunikativen Raum: Neun Anhaltspunkte“. In: Bernsen, Michael/Eggert, Elmar/Schrott, Angela (eds.): Historische Sprachwissenschaft als philologische Kulturwissenschaft. Festschrift für Franz Lebsanft zum 60. Geburtstag. Göttingen, V\&R unipress: 393-404.

Labov, William (2001): Principles of linguistic change. Band 2. Social factors. Malden, MA/Oxford: Blackwell. (= Language in Society 29).

Lehmann, Christian (1995): Thoughts on grammaticalization. 3rd edition. Berlin: Language Science Press. (= Classics in Linguistics 1).

Lenz, Alexandra N. (2016): „Konjunktiv (würde/täte)“. In: SyHD-atlas. syhd.info/apps/ atlas/\#konjunktiv-wuerde-taete [27.05.2021].

Lenz, Alexandra N. (2019): „Bairisch und Alemannisch in Österreich“. In: Herrgen, Joachim/Schmidt, Jürgen E. (eds.): Sprache und Raum - Ein internationales Handbuch der Sprachvariation. Band 4: Deutsch. Berlin/Boston, de Gruyter Mouton: 318-363. (= Handbücher zur Sprach- und Kommunikationswissenschaft 30.4).

Lenzhofer, Melanie (2017): Jugendkommunikation und Dialekt. Syntax gesprochener Sprache bei Jugendlichen in Osttirol. Berlin/Boston: de Gruyter. (=Empirische Linguistik/Empirical Linguistics 6).

Lotze, Stefan/Gallmann, Peter (2009): „Norm und Variation beim Konjunktiv II“. In: Konopka, Marek/Strecker, Bruno (eds.): Deutsche Grammatik - Regeln, Normen, Sprachgebrauch. Berlin/New York, de Gruyter: 222-239. (= Jahrbuch des Instituts für deutsche Sprache 2008). 
Löw, Martina/Steets, Silke/Stoetzer, Sergej (2008): Einführung in die Stadt- und Raumsoziologie. 2., aktual. Aufl. Opladen/Farmington Hills: Budrich.

Mattheier, Klaus J. (1982): „Sprachgebrauch und Urbanisierung. Sprachveränderungen in kleinen Gemeinden im Umfeld großer Städte“. In: Bausch, Karl-Heinz (ed.): Mehrsprachigkeit in der Stadtregion. Jahrbuch 1981 des Instituts für deutsche Sprache. Düsseldorf, Schwann: 87-107. (= Sprache der Gegenwart 56).

Merkle, Ludwig (1993): Bairische Grammatik. 5. Aufl. München: Hugendubel.

Moosmüller, Sylvia (1991): Hochsprache und Dialekt in Österreich. Soziophonologische Untersuchungen zu ihrer Abgrenzung in Wien, Graz, Salzburg und Innsbruck. Wien/Köln/Weimar: Böhlau.

Moosmüller, Sylvia/Scheutz, Hannes (2013): „Chain shifts revisited: The case of monophthongisation and econfusion in the city dialects of Salzburg and Vienna“. In: Auer, Peter/Reina, Javier Caro/Kaufmann, Göz (eds.): Language variation - European Perspectives 4. Amsterdam, Benjamins: 173-186.

Mortelmans, Tanja/Smirnova, Elena (2010): „Plusquamperfektkonstruktionen mit Modalverb im Deutschen“. In: Bittner, Dagmar/Gaeta, Livio (eds.): Kodierungstechniken im Wandel: Das Zusammenspiel von Analytik und Synthese im Gegenwartsdeutschen. Berlin/New York, de Gruyter: 47-66. (=Linguistik - Impulse \& Tendenzen 34).

Nübling, Damaris (1997): „Der alemannische Konjunktiv II zwischen Morphologie und Syntax. Zur Neuordnung des Konjunktivsystems nach dem Präteritumschwund“. In: Ruoff, Arno/Löffelad, Peter (eds.): Syntax und Stilistik der Alltagssprache. Beiträge der 12. Arbeitstagung zur Alemannischen Dialektologie, 25. bis 29. September 1996 in Ellwangen/Jagst. Tübingen, Niemeyer: 107-121. (=Idiomatica 18).

Pickl, Simon (2022): ,Formen und Funktionen des Konjunktivs II in historischen ostoberdeutschen Predigten. Eine Langzeit-Perspektive“. Linguistik Online: in diesem Band.

REDE (2008-): Regionalsprache.de (REDE). Forschungsplattform zu den modernen Regionalsprachen des Deutschen. Herausgegeben von Jürgen Erich Schmidt, Joachim Herrgen und Roland Kehrein. Bearbeitet von Dennis Bock, Brigitte Ganswindt, Heiko Girnth, Simon Kasper, Roland Kehrein, Alfred Lameli, Slawomir Messner, Christoph Purschke, Anna Wolańska. Marburg, Forschungszentrum Deutscher Sprachatlas.

Schnelzer, Klaus Otto (2008): Konjunktiv kontrastiv. Zur Morphologie bairischer und isländischer Verben. Regensburg: vulpes. (= Regensburger Dialektforum 11).

Selting, Margret et al. (2009): „Gesprächsanalytisches Transkriptionssystem 2 (GAT 2)“. Gesprächsforschung - Online-Zeitschrift zur verbalen Interaktion 10, 353-402. gespraechsforschung-ozs.de/heft2009/px-gat2.pdf [27.05.2021].

Smakman, Dick/Heinrich, Patrick (2017): „Introduction: Why Cities Matter for a Globalising Sociolinguistics“. In: Smakman, Dick/Heinrich, Patrick (eds.): Urban Sociolinguistics. The City as a Linguistic Process and Experience. London/New York, Routledge: 1-11.

Smirnova, Elena (2006): Die Entwicklung der Konstruktion würde + Infinitiv im Deutschen. Eine funktional-semantische Analyse unter besonderer Berücksichtigung sprachhistorischer Aspekte. Berlin/New York: de Gruyter. (= Studia Linguistica Germanica 82).

Soukup, Barbara (2009): Dialect use as interaction strategy. A sociolinguistic study of contextualization, speech perception, and language attitudes in Austria. Dissertation. Georgetown University. Washington D.C. 
Soukup, Barbara/Moosmüller, Sylvia (2011): „Standard language in Austria“. In: Coupland, Nikolas/Kristiansen, Tore (eds.): Standard Languages and Language Standards in a Changing Europe. Oslo, Novus: 39-46.

Stöckle, Philipp (2020): „Dialektvariation an der Schnittstelle von Syntax, Morphologie und Lexik. Der Konjunktiv II in den bairischen Dialekten Österreichs und Südtirols“. In: Christen, Helen et al. (eds.): Regiolekt - Der neue Dialekt? Akten des 6. Kongresses der Internationalen Gesellschaft für Dialektologie des Deutschen (IGDD). Stuttgart, Steiner: 149-173. (=Zeitschrift für Dialektologie und Linguistik, Beihefte 182).

Stein, Stephan (1995): Formelhafte Sprache. Untersuchungen zu ihren pragmatischen und kognitiven Funktionen im gegenwärtigen Deutsch. Frankfurt a. M./Wien: Lang. (=Sprache in der Gesellschaft 22).

Taeldeman, Johan (2005): „The influence of urban centres on the spatial diffusion of dialect phenomena“. In: Auer, Peter/Hinskens, Frans/Kerswill, Paul (eds.): Dialect change. Convergence and Divergence in European Languages. Cambridge, CUP: 263-283.

Thieroff, Rolf (2010): „Mood in German“. In: Rothstein, Björn/Thieroff, Rolf (eds.): Mood in the Languages of Europe. Amsterdam/Philadelphia, Benjamins: 133-154. (= Studies in Language Companion Series 120).

Vergeiner, Philip C./Bülow, Lars (2022): „Der Konjunktiv II in den ruralen Basisdialekten Österreichs. Quantitative und qualitative Befunde“. Linguistik Online: in diesem Band.

Wandruszka, Mario (1979): Die Mehrsprachigkeit des Menschen. München: Piper.

Wiesinger, Peter (1989): Die Flexionsmorphologie des Verbums im Bairischen. Wien: Verlag der österreichischen Akademie der Wissenschaften. (= Philosophisch-historische Klasse, Sitzungsberichte 523).

Zehetner, Ludwig (1978): „Kontrastive Morphologie. Bairisch/Einheitssprache“. In: Ammon, Ulrich/Knoop, Ulrich/Radtke, Ingulf (eds.): Grundlagen einer dialektorientierten Sprachdidaktik. Theoretische und empirische Beiträge zu einem vernachlässigten Schulproblem. Weinheim/Basel, Beltz: 313-331.

Ziegler, Arne/Glantschnig, Melanie (2013): „Jugendsprache(n) und Grammatikunterricht. Blinde Flecken auf der Landkarte - Jugendsprachforschung in Österreich als Chance für die Schulgrammatik?“ In: Köpcke, Klaus-Michael/Ziegler, Arne (eds.): Schulgrammatik und Sprachunterricht im Wandel. Berlin/Boston, de Gruyter: 287-306. (= Reihe Germanistische Linguistik 297).

Ziegler, Arne/Oberdorfer, Georg/Herbert, Kristina (in print): „Diminutives and their variation in spoken interaction in urban areas - The Austrian case“. In: Ziegler, Arne/Edler, Stefanie/Oberdorfer, Georg (eds.): Urban Matters. Current Approaches in Variationist Sociolinguistics. Amsterdam/Philadelphia, Benjamins. (= Studies in Language Variation).

\section{Internetquellen}

Statistik Austria $1=$ Statistik Austria (Stand 01.01.2020): statistik.at/blickgem/pr2/g90001.pdf bzw. statistik.at/blickgem/pr2/g60101.pdf [22.11.2020].

Statistik Austria 2 = Statistik Austria (Stand 01.01.2020): statistik.at/blickgem/index [22.11.2020].

REDE = regionalsprache.de, im vorliegenden Fall: regionalsprache.de/atlanten-undkarten.aspx [27.05.2021] 
Sprachatlas von Niederbayern (SNiB): https://www.phil.uni-passau.de/deutsche-sprachwis senschaft/forschung/sprachraumforschung/snib [02.02.2022].

SprachGIS = regionalsprache.de/SprachGIS/Map.aspx [27.05.2021]

\section{Korpora}

ULA-Korpus $=$ Urban-Language-in-Austria-Korpus, erarbeitet/aufgebaut im Forschungsprojekt Wien und Graz - Städte und ihre sprachlichen Strahlkräfte 